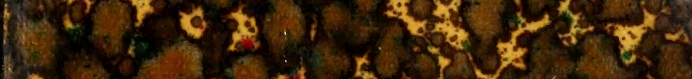

C $\cos ^{5} 5^{5} \cdot 2^{2}$

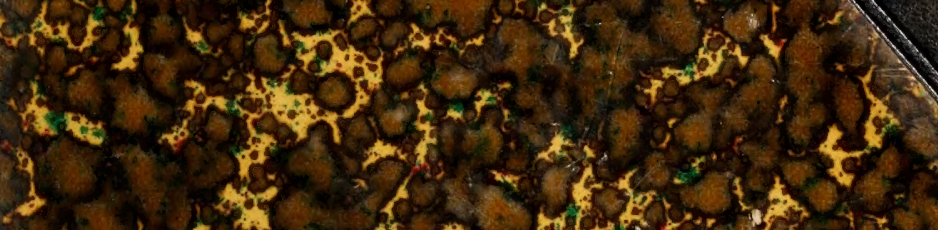

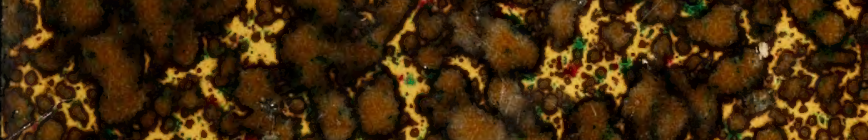

xotion

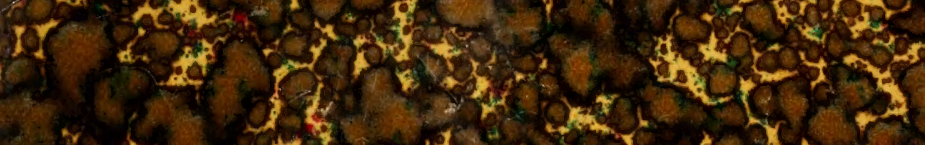

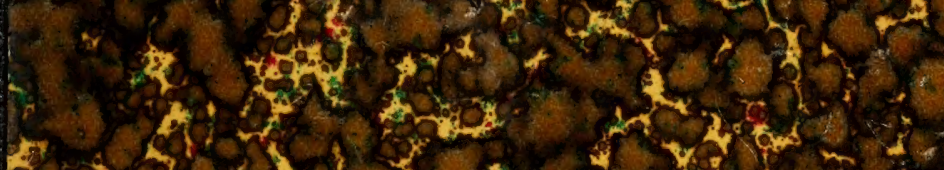

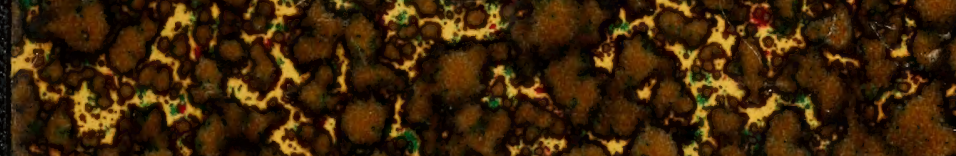

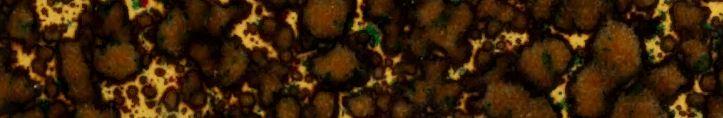

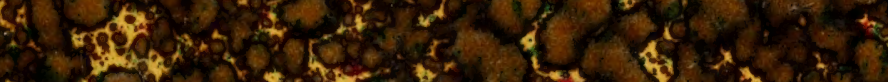

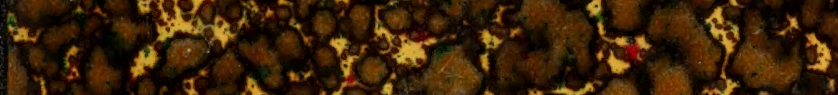

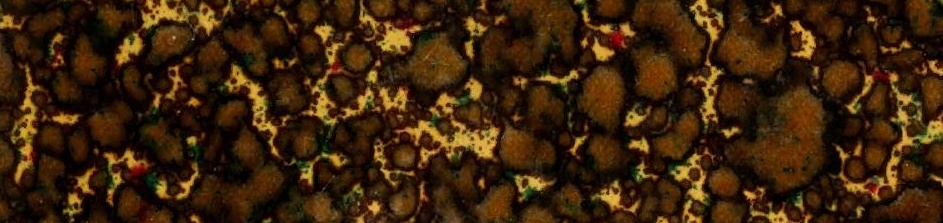
1.

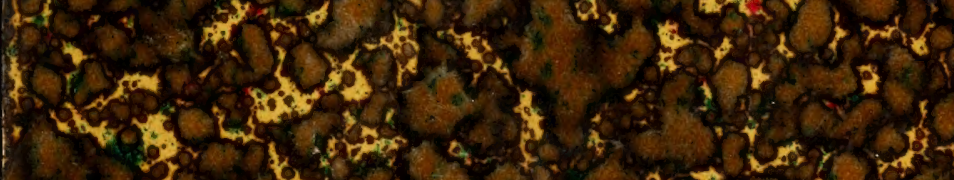

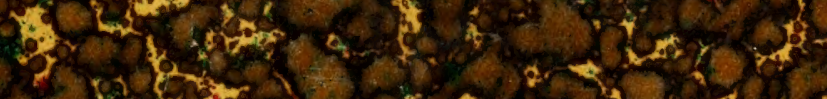

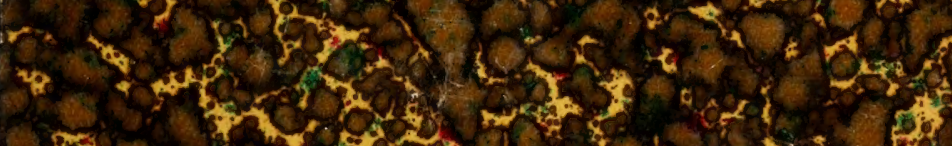

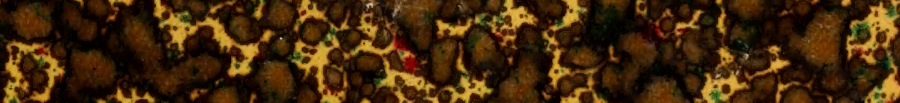

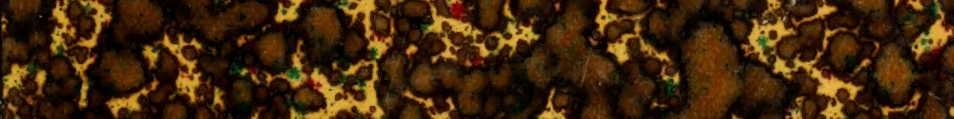

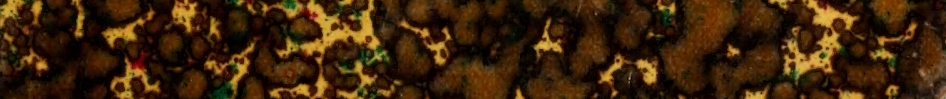

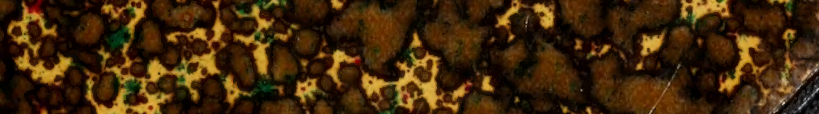

B. 30 .

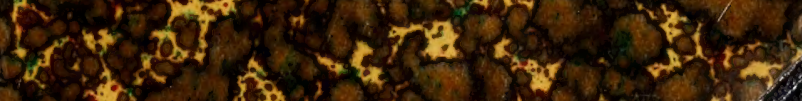

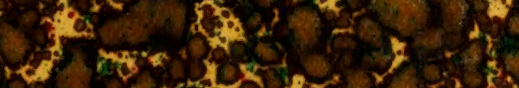

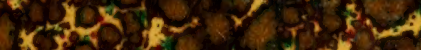




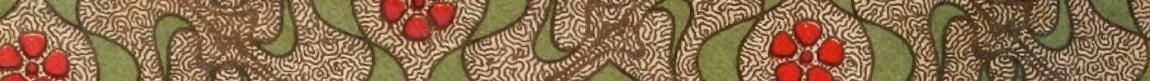

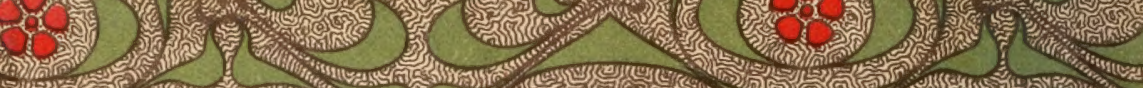

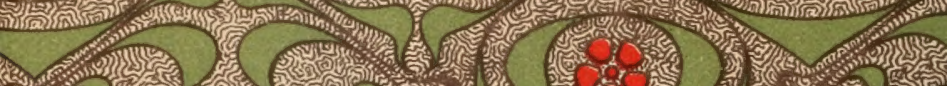

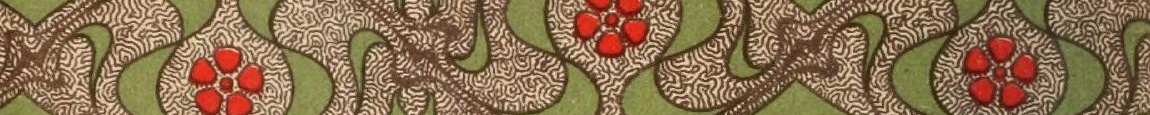

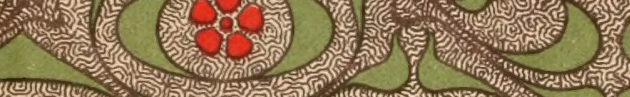

m. n n n $\sqrt{8}$ 23 12 ( )

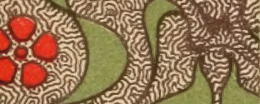
ins

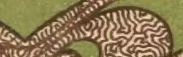
( 180 (2)

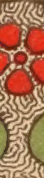

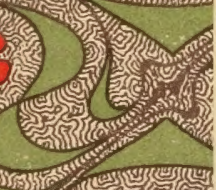
(3)

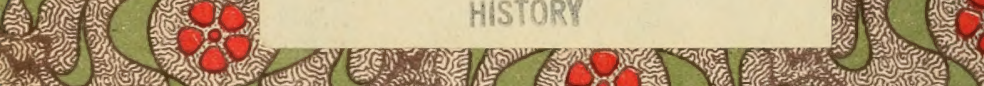

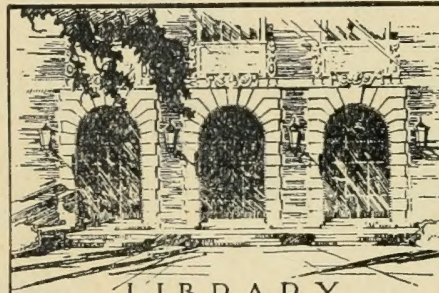

L I B R A R Y OF THE

UN IVER S ITY

OF ILLINOIS

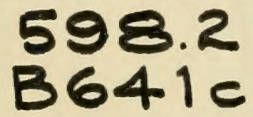

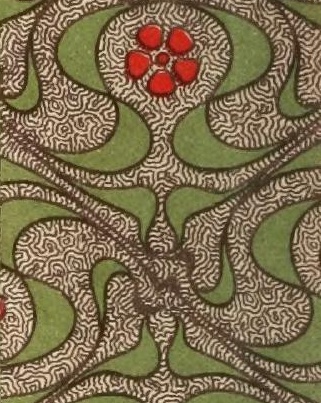

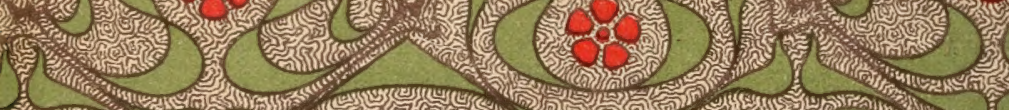

NATURALS

HISTOOM
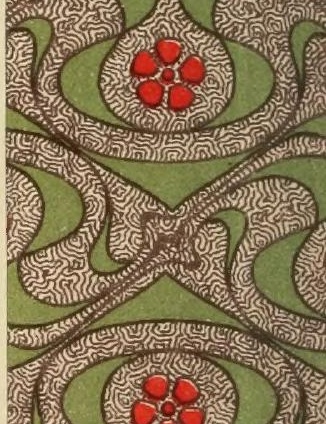
(n) 5.1. 2105 INin (10.0) 150. 
Digitized by the Internet Archive in 2010 with funding from

University of Illinois Urbana-Champaign

http://www.archive.org/details/catalogomtodico00bona 
Auretererventin. 



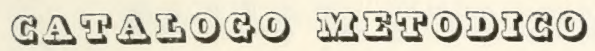

D E G L I

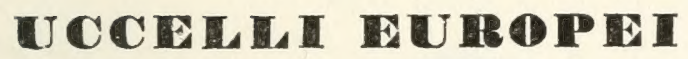





\title{
CATAlOgO METODICO
}

\author{
degli
}

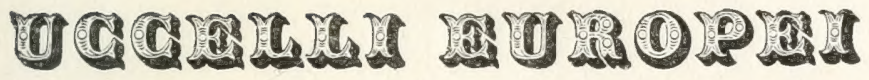

$\mathscr{D}_{i}$

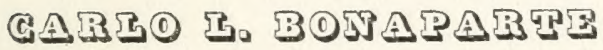

PRINCIPE DI CANINO

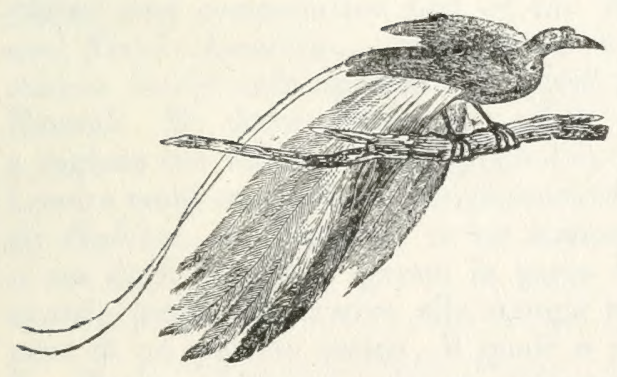

BOLOGN A

PEI TIPI DI JACOPO MARSIGLI

1842 
Dagli Annali delle Scienze Naturali T. VIII.

2. semestre 1842 . 
Carissimo Alessandrini

Quando nel r 838 mi pregiai mandarvi, come è sempre delle coserelle che vado pubblicando, la mia , Geographical and comparative List of the Birds of Europe and North-America,, voi mi deste un segno di vera amicizia desiderando riprodurla in cotesti Annali di Scienze Naturali. Mi dolse dovessi per allora tenermi al niego a cagione che voleva innanzi portare in quella edizione di Londra molti cangiamenti e miglioramenti, soprattutto negli Habitat, che frequenti ve ne mancava, e persino ve n' era degli sbagliati. Questo in parte avvenne, che non avendo potuto io assistere alla stampa mi riposi nel sapere di un provato amico, il quale o non potè o non fu sollecito adoperarsi a tenore delle mie istruzioni. Siccome però $\mathrm{i}$ vostri desiderî mi sono comandamenti, e me stesso regalo ogni volta possa offrire a voi, ho sempre inteso l'animo e lo studio all' antica promessa, di che vengo ora a sdebitarmi in parte mandandovi il Catalogo già pronto degli Uccelli d'Europa. Potete bene antivedere, che per tal lungo tempo, di molte cose migliorate o nuove sarà fornito questo lavoro, $\mathrm{e}$ vie più che a 
stendere di fresco la Introduzione alla Classe Uccelli della mia Fauna, mi es stato mestieri ristudiare con assai attenzione la intera Ornitologia massimamente di Europa. Non tacerò che mi ha valuto non poco utilmente il bel libro del Keyserling e del Blasius sui Manmiferi e gli Uccelli Europei. Ho contrasseguato di asterisco tutte le specie italiane onde si avesse doppio Catalogo utilissimo a una volta tanto alla Ornitologia della sola Penisola, quanto a quella di Europa intiera.

Senza molto andare in lungo vi rimetterò eziandio la seconda edizione del Catalogo degli Úccelli americani per servire di confronto, cui tardo a dare, desideroso arricchirla di quei del Messico. Alla redazione di siffatte liste per ogni parte di mondo non mancherò stimolare l' opera dei più valenti, perchè facilissimamente si verrebbe per via di quelle nella intrapresa di una Ornitologia generale, della quale come meglio sappia andrò io stesso occupandomi. Il mio Sistema Arius avendo subito non leggieri mutamenti dalla sua pubblicazione tho creduto beue preporne rettificatamente lo scheletro a questa Lista. Vedrete che delle sole Famiglie e Sottofamiglie che non caratterizzai nel Tol. III. p. 440 . di cotesto Giornale stesso, do qui i caratteri; e ciò per non allungare in ripetizioni; cosi pure ho tralasciato altre minori rettificazioni, che avrei bene introdotte se tutto si fosse dovuto ristampare il Sistema. Il numero 62 delle Famiglic è oggi asceso a 7o, mercè l'adozione di quella dei Todidi collocata, fra' Coraciadidi e i Prionitidi, la separazione dei Promeropidi dagli Upupidi, e la formazione di quella dei malamente dispersi Mlenuridi racchindenti tre distinte Sottofamiglie, Menurini, Malurini, Troglodytini, nei Passeri; di quella dei Talegallidi nelle Galline; dei Dididi, degli Apterigidi negli Struzzr e dei Palamedcidi nelle Gralle. E le r5o Sottofamiglie, già salite a 155 in una più recente edizione, sono ora pervenute a 180 . Se i numeri non si succedono progressivi in esse Famiglie e Sottofamiglie dipende dalI' intervallo delle intralasciate non Europee. I pochi can- 
giamenti nella nomenclatura, come per es. il genere Tinnunculus, Vieill. invece di Cerchneis, Boie, e la specie Phaenicoplerus roseus, Pallas, invece di Ph. antiquorum Temm. sono da attribuire ad avere riconosciuto posteriormente alla prima ediziouc essere legittimo per anteriorità il nome sostituito .

Se voi, cultore dottissimo delle cose naturali, questo giudicherete buon seme che frutti vantaggio alla Scienza, io ve ne verò somministrando per le altre tre Classi, Mammiferi, Amfibi, Pesci. La prima, non meno che questa degli Uccelli, aveva già in pronto gran tempo innanzi potessi giovarmi a perfezionarle di recenti lavori, i quali a dir vero non mi scemarono la fatica del comporre questi Cataloghi, ma soltanto valsero a verificazione. Sto pur preparando una seconda edizione delIa Lista Generi delle quattro Classi, aggiuntevi le rispettive specie tipiche, Prodromo necessario al mio Srstema Ventebratorua ; della quale a mano a mano riceverete. Fatevi certo che terrò sempre la mente a discolparmi dell' obbligante rimprovero abbia io trasandato il vostro Giornale, e mostrare anche per tal modo, che se l'amore che porto agli studi naturali mi occupa ad apprenderlo maggiormente dai Professori italiani o per corrispondenze divise, $o$ in Riunione scientifica congregati, non trascuro affatto la mia Zoologia. Questo vi dichiarerò eziandio che mi è sempre dolce manifestarmi sollecito di quella Bologna, ove ben voleste voi rimanervi, non cedendo, con raro esempio, agli allettamenti di miglior fortuna che si porgevano al conosciutissimo vostro merito.

In quelli che veracemente lo ammirano abbiatemi sempre

Firenze 5 Aprile 1842.

affezionatissimo e obbligatissimo

Carlo L. Bonaparte

Principe di Canino. 


\section{SPECCHIO GENRRAL}

$D E L$

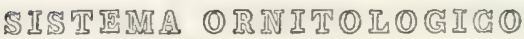 \\ CIISTIS : AVI}

SUBCLASSIS I. INSESSORES

ORDO I. PSITTACI

FAMILIAE

SUBFAMILIAE

1. Psittacidae.

I. Macrocercinae.

2. Psittacinae.

3. Platycercinae (a).

4. Pezoporinae.

5. Lorinae.

6. Plyctolophinae.

\section{ORDO 2. ACCIPITRES}

*2. Volturinae.

${ }^{*}$ ๆ. Vulturinae.

8. Cathartinae (b).

*9. Gypaetinae.

1 o. Gypohieracinae (c).

3. Gypogeranidae. I1. Gypogeraninae 
9

"4. Falconidae.

12. Polyborinae .

${ }^{*}$ I3. Aquilinae .

${ }^{*}$ 14. Buteoninae.

${ }^{*}$ I5. Milvinae .

${ }^{*} 6$. Falconinae .

${ }^{*}{ }^{*} 7$. Accipitrinae.

* 8 . Circinae.

*5. Strigidae.

${ }^{*}$ I9. Surninae .

${ }^{*}$ 20. Buboninae .

${ }^{*}$ I. Ululinae.

*22. Striginae.

\section{ORDO 3. PASSERES.}

\section{Iribus 1. Ambulntores}

${ }^{*} 6$. Caprimulgidae.

23. Podarginae.

${ }^{*}$ \%. Caprimulginae.

*25. Scotornithinae.

*7. Crypelidale.

${ }_{2}^{*} 6$. Cypselinae.

*8. Hirundinidae

${ }^{*}$ 27. Hirundininae.

*9. Coraciadidae.

${ }^{*} 28$. Oriolinae.

*29. Coraciadinae.

3o. Steatornithinae (d).

io. Tomibae. (A)

3 ז. Eurylaeminae.

32. Todinae (e).

II. Prionitidae

33. Prionitinae.

*12. Alcedinidae.

34. Dacelininae.

$\star 35$. Alcedininae. 
* 13. Meropidae.

* 1 4. Upupidae.

I5. Promeropidae.(B)

16. Paradiseidae.

17. 'Trochilidae.

18. Cherebidae. (C)

19. Melliphagidae.

*20. Menuridae. (D)

*21. Certhidae.

*22. Paridae.

23. TaNagridae.

24. Alaudidae.
*36. Meropinae.

${ }^{*} 37$. Upupinae.

38. Promeropinae. (f)

39. Paradiseinae.

40. Trochilinae.

4I. Phaetornithinae (g).

42. Lampornithinae (h).

43. Nectarininae.

44. Caerebinae.

45. Mellithreptinae (i).

46. Melliphaginae. (k)

47. Myzomelinae (1).

48. Menurinae (m).

49. Malurinae (n).

*5o. Troglodytinac (o).

*5x. Certhinae.

*52. Tichodrominae.

53. Furnarinae (p).

54. Anabatinae.

55. Dendrocolaptinae (q).

*56. Sittinae.

*57. Parinae.

58. Piprinae.

59. Tanagrinae.

6o. Pytilinae ( $r$ ).

6 I. Phytotominae.

*62. Alaudinae. 
*25. Motacillidae.

${ }^{*}$ 6. Turdidae.

*27. Muscicapidae.

"28. Aypelididae.

*29. Lamidae.
11

*63. Anthinae (s).

${ }^{*} 64$. Motacillinae.

${ }^{*} 65$. Cinclirae.

66. Myiotherinae .

67. Timalinae.

*68. Ixodinae.

${ }^{x} 69$. Turdinae.

* go. Calamoherpinae.

${ }^{*} 7$ r. Sylvinae.

*72. Saxicolinae.

73. Sylvicolinae.

${ }^{*} 74$. Muscicapinae.

75. Taeniopterinae.

76. Tyranninae.

77. Psaridinae.

78. Vireoninae.

79. Liothricinae.

*8o. Ampelidinae.

$8 \mathrm{r}$. Cotinginae.

82. Gymnoderinae.

83. Ceblepyrinae.

84. Edolinae.

*85. Laninae.

86. Tamnophilinae.

87. Baritinae. 
*3o. Corvidae.

*31. Fringillidae.

33. Colidae.

33. Bugerontidae.

\section{(1) ributs 2, Scrusares.}

34. Ramphastidae.

*35. Cuculidae.

*36. Picidae.
${ }^{*} 88$. Garrulinae.

89. Glaucopinae.

*9o. Corvinae.

9 r. Coracininae.

*92. Sturninae.

93. Lamprotornithinae.

94. Quiscalinae.

95 . Icterinae.

96. Ploceinae.

*97. Emberizinae.

98. Geospizinae ( $\mathrm{t}$ ).

*99. Fringillinae.

${ }^{*}$ 1 0o. Loxinae.

I о . Colinae.

I02. Bucerontinae.

ı 3. Ramphastidinae.

ro4. Saurotherinae.

1 05. Phaenicophaeinae (u).

ro6. Crotophaginae.

107. Indicatorinae.

1 08. Centropinae (v).

* 1 09. Cuculinae.

I 1 o. Coccyzinae.

* r r. Yunginae.

I I2. Colaptidinac (x).

* 13. Picinae.

I 14 . Picumninae. (y). 
37. Bucconidar.

38. Capitonidae.

39. Galbulidae.

40. 'Trogonidae.

41. Musopharidal.
I 5 . Bucconinae.

ェ 6. Capitoninae .

1 7. Galbulinae.

118. Trogoninae.

I I9. Musophaginae.

120. Opisthocominae (z).

\section{ORDO 4. COLUMBAE.}

*/3. Columbidal.

121. Treroninae (aa).

*122. Columbinae.

ז23. Gourinae (bb).

\section{SUBCLASSIS II. GHALLA'IORES。}

\section{ORDO 5. GALLINAE.}

* 4 3. Pteroclidae.

4伯. Chionididae.

45. Megapodidae. (E)

46. Penelopidae.

*47. Pihasianidae.
124. Syrrhaptinae.

* 125 . Pteroclinae.

126. Thinocorinae.

127. Chioninae.

I28. Talegallinae (cc).

I 29. Megapodinae (dd).

13o. Penelopinae.

131. Cracinae (ce).

132. Pavoninae.

* 133 . Phasianinae. 
*48. Tetraonidae. ${ }^{*}$ i34. Tetraoninae.

${ }^{*}$ I 35 . Perdicinae.

${ }^{*}$ I 36 . Turnicinae.

49. Crupturidae. $\quad$ 37. Crypturinae.

\section{ORDO 6. STRUTHIONES.}

50. Struthionidae.

5r. Dididae. (F)

52. Apterygidae. (G) 140. Apteryginae,
I38. Struthioninae.

139. Didinae.

\section{đributs 1. Gallinaren.}

*53. Charadridae.

*54. Scolopacidae.

*55. Recurvirostridae

*56. Phalaropodidae.

${ }^{*} 57$. Rallidae.

58. Palamedeidae.(H)

*59. Psophidae.

${ }^{*}$ I 4 I. Otidinae.

${ }^{*}$ I 42. Charadrinae.

${ }^{*}$ I 43. Vanellinae (ff).

${ }^{*}$ I 44. Haematopodisae.

${ }^{*}$ × 45 . Scolopacinae.

${ }^{*}{ }^{*}$ 46. Tringinae.

${ }^{*}$ 4 47 . Recurvirostrinae.

* 48. Phalaropodinae.

* I f9. Rallinae.

150. Araminae (gg).

I5 ז. Parrinae.

152. Palamedeinae.

I53. Psophinae (hb).

${ }^{*}$ 5 54. Gruinae. 


\section{Tribus 2. Ansseracent.}

*6o. Ardeidae.

*61. Tantalidae.

*62. Plataleidae. (I)

*63. Phaenicopteridae.
${ }^{*}$ 55. Ardeinae.

* 56 . Ciconinae.

157 . Cancrominae.

I58. Dromadinae (ii).

${ }^{*} 59$. Tantalinae.

*6o. Plataleinae.

${ }^{*}$ 161. Phaenicopterinae.

\section{ORDO 8. ANSERES.}

${ }^{*} 64$. Anatidae.

*162. Cygninae.

${ }^{*}$ 163. Anserinae.

${ }^{*}$ 64. Anatinae.

${ }^{*}$ 165. Fuligulinae.

*166. Merginae.

*65. Pelecanidae.

*66. Laridae. I67. Heliornithinae (kk). 168. Plotinae.

${ }^{*}$ 69 . Pelecaninae.

* э o. Sterninae.

171. Rhynchopinae.

${ }^{*}$ I 2. Larinae.

${ }^{*}$ I 7 . Lestrinae.

*67. Proceltaridae. $\quad$ I74. Procellarinae. 
*68. Colymbidae.

${ }^{*}{ }_{175}$. Podicipinae.

${ }^{*}$ i 6 . Colymbinae.

${ }^{*}$ I 77 . Urinae.

*69. Alcidae. I 78 . Phaleridinae.

${ }^{*}$ 1 79. Alcinae.

70. Spheniscidae. 180. Spheniscinae.

* Familiae Eur. 43.

* Subfamiliae Eur. 82. 


\section{I I X}

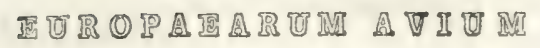

EUBCLASTIS I. INSESSORES

ORDO II. ACCIPITRES

Familia 2. Vulturidae

Subfamilia 7. Vulturinae.

Gevos I. Neopurox, Savg. Perenopterus, Cuv.

1. N. pergnopterus, Savg. Vultur percnopterus, L. Gould tab. 3.

\section{GYPS, Savg.}

*2. G. Fulvus, G. Gray, Vultur fulvus, Br. V. leucocephalus, Meyer, Gould tab. I.

*3. G. vulgaris, Savg. $V$. percnopterus, Pall.

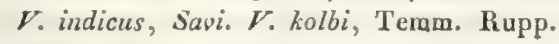
Allas. tab. 32, nec Auct.

\section{Aegrpius, Savg.}

"4. A. cineneus, Bp. Vultur cinereus, L. Eur.m.or. Afric. Aegrpius niger, Sarg. Gould tab. 2.
Calidior. Orbis antiq.

Eur. occ.m.Afr. Ital. rar.

Sardin. Eur. or. Afr. As. occ. 
4. Veltur, L. Otogyps, Gr.

*5. V. Auricularis, Daud. $\boldsymbol{V}$. aegyptius s. Graecia, Afric. Oricou, Temm. 1. col. $407 . \quad$ Italia accid.

\section{Subfamilia 9. Gypaetinae.}

5. Grpatetus, Ray. Phene, Savg.

*6 G. banbatus, Cur. $\boldsymbol{V}$. barbatus, L. P/l. Pyraen. Alp. Siossifraga, Savg. Gould t. 4 .

a. occidentalis, major obscurior.

$\beta$. orientalis, minor magis fulvus.

bir. Sard. Pedem.

Alp. Eur. As. Afr. Sard. Pyraen, optima sp. distinctissimus a G. meridionali Afr.

\section{Familia 4. Falconidae.}

Subf. 13. Aquilinae.

6. AQuILA, Briss.

*7. A. Furva, Sarg. F. clurysaetos et fulvus, L.

Europ. univers, A. nobilis, Pall. Gould. tab. 6.

As. s. Am.

*8. A. нецtaca, Savg. F. Imperialis, Bechst. Eur. m. Afr. A. chrysaetos, Leisl. Gould. Lab. 5.

*9. A. vaevia, Br. F. naevius et maculatus, Gm., A. clanga, Pall. Gould tab. 8.

Eur. or. Sibir. acc. Ital.

*io. A. bonellit , Bp. F. bonelli, Temm. Gould Sard. Gallia m. tab. 7 .

Afr. s. As.

rr. A. pennata, Cup. $F$. pennatus, Gmel. Eur. m. ad or. Gould tab. 9. Africa. 


\section{Haluetes, Savg.}

*2. H. Albicilda, Savg. Falco albicilla et os- Eur. univ. As. s, sifragus, Gm. Gould t. ro.

13. H. Leucocephalus, Savg. Falco leucocephalus, L. Gould t. II.

Am. s. Norvegia, quatenus var. 12. pro am. avi non habeatur.

14. H. Levcorypha, K. et Bl. Aquila leucorypha, Pall.

Eur. or. As, occ. ad mare Casp.

\section{Paxdion, Savg.}

*5. P. inaliatedes, Cuv. Falco haliaetus, L. Tot. antiq. orb. P. fluviatilis, Savg. Gould t. 12 .

\section{Cincaetus; Vieill.}

*16. C. Galurcus, Vieill. Falco gallicus, Gm, Eur. m. et med. Aq. braclidactyla, Mey. et W. Gould t. 13 .

17. C. Hypoleucos, $\boldsymbol{K}$. et Bl. Accipiter hypoleucos, Pall.

Junior praecedentis?

\section{Subfamilia I4. Buteoninae.}

10. Butaetes, Less. Archibuteo, Brehm.

*18. B. LAgopes, Bp. Falco lagopus, L. Buteo lagopus, K. et Bl. Gould t. 15.

II. Buteo, Bechst.

"ig B. vulganis, Bechst. F. butée, L. B. mutans et fasciatus, Vieill. B. pojana, Savi, Gould t. 1千́
Eur, univ. As. oce. Afr. 
12. Pensis, Cuv.

*20. P. Aprvonus, Cuv. Falco apivorus, L. Eur. univ. As. Acc. lacertarius, Pall. Gould t. 16. occ. Ital. migr.

\section{Subfamilia i5. Milvinae.}

13. Murves, Beelista

*21. M. Niger, Br. F. ater et aegyptius, Gm. Eur.or. Sib.As, Acc. milves, Pall. Gould 1. 29. centr. Afr.

*22 MI. regalis, Br. Falco milous, L. Mil- Eur. med.et m, vus ictinus, Sarg. Gould t. 28.

\section{Niuccends, Vige.}

23. N. Funcatus, Vig. Falco furcatus, L. Americas.accid, Gould 1. 30 . Anglia.

15. Elanus, Leach, Elanoides, Vieill.

44. E. yelanopterus, Leach, Falco melanopterus, Daud. Gould t. 3 I.

Afr. acc, Eur. c.

Subfamilia 16. Falconinae,

16. FAlco, L.

a. Hierofalco, Cur.

25. F. gyaflco, L. Falco islandicus, Br. Russia s. Scand, Hancock, Gould t. Ig.

Scot., Island.

26. F. candicass, Gm. $F$. groenlandicus, Han. Groenl, Sibiria.

b. Falco. Cuv. Rhyncodon, Nitzsch.

27. F. Laxanius, L. Lanarius, Gesn. F. la- Eur. m. ad or. niarius, Pall. Gould t. $20 . \quad$ As. occ.

28. F. praegrines, L. F. peregrinus, Br. Eur. univ. As. Gould t, 21. 
c. Lithofalco, Bp.

*29. F. aesaloo, Gm. F. lithofalco, Gm. Eur. unis. A3. F. regulus, Pall. Gould t. 24 . Afr.

d. Dendrofalco, Br. Hypotriorchis, Bois.

*3o. F. suвbuten, L. Gould t. 23.

'Tot. orb. antiq.

3r. F. Concolor, Temm. F. ardosiaceus?

Africus s, acc.

Vieill, Gould tab. 25.

Eur. m.

*33. F. eleonorae, Genè, Bp. Icon. I. t. I.Av. Sardinia, Afric.

As, occ.

e. Erytliropus, Brehm. Panngchistes, Kaup.

*33. F. vespertinus, L. F. rufipes, Beseclie. Eur. orient. m. Gould t. 23.

As. occ.

17. Tinnunculus, Bi. Gerchneis, Boie.

*34. T. crinchris, Bp. F. tinnunculoides, Nalt. Eur, m. Afr.

$F$. cenchris, Naum. Gould t. 27.

*35. T. aladdarius, Br. F. tinnunculles, L. Eur. unir. As.

Cerchn. tinnunculus, Boie, Gould t. $26 . \quad$ Afr.

\section{Subfamilia I 7. Accipitrinae.}

18. Accipiter, Ray. Sparvius, Vieill.

*36. A. visus, Pall. Falco nisus, L. Acc. fringillarius, Ray. Gould. t. 18.

Eur. univ. As.

19. Astur, Bechst Dazdalion, Savg.

37. A. palumbarius, Gm. F.palumbarius, L. Eur, univ. As,s. Gould. t. 17 .

Subfamilia 18. Circinae.

20. Cincus, Br.

a. Circus, Bechst.

38. C. Aerugrosus, Bp. Falco aeruginosus, L. Eur. unir. As. et rufus, Gm. Gould t. 33 .

Afr. 
b. Strigiceps, Bp.

*39. C. cyanets, Bechst. F. cyaneus, L. Eur. univ. As. Acc. variabilis, Pall. Pygargus, Belon. s. et or. G. t. 33 .

*4o. C. swansonr, Smith, C. pallidus, Sykes, Eur. med. et m. F. dalmatinus, Rüpp. Gould t. 34. Afr. As.

*/4r. C. cineraceds, Montag. C. montagui, Eur. As. Afr. Vieill. F. pygargus? L. Gould t. 35.

Familia 5. Strigidae.

Subfamilia 19. Suminae.

21. Sunvia, Dum.

*42. S. vuula, Bp. Strix ulula, L. S. funerea, Lath. Gould t. 45 .

Eur. s, et med.

Sib. Am. s.

\section{Nyctea, Steph.}

43. N. CaNdida, Bp. Str. nyctea, L.S.cant Eur. As. Am. s. dida, Lath. Gould t. 43.

\section{3- Gladcidiur, Boie.}

44. G. rasserinum, Boie, S. passerina, L. Eur. s. ad or. S. acadica, Temm. Gould t. 50 .

24. ATHene, Boie. Nyctipetes, $S w$.

*45. A. noctua, Boie, S. noctua, Retz. $S$. Eur. m. et med. passerina, Temm. Gould t. 48 .

As. occ.

25. Scops, Savg. Ephialtes, K. et Bt.

*46. S. zorca, Bp. S. scops, L. S. aldrovandi, Ray. S. ephialtes, Savg. G.t. 4I.

Eur. m. et centr. Afr. 


\section{Subfamilia 20. Buboninae.}

26. Вuвo, Cuv. Heliaptex, Sw.

a. Ascalaphia, Is. G.

*47. B. ascalaphus, Sarg. Strix ascalapluzs, Temm. Asc. savigny, Is. G. Gould t. 38.

Afr. Eur. or.acc.

b. Bubo, Sibb.

*48. B. maximus, Sibb. S. bubo, L. Bubo Eur. univ. As. atheniensis, Boie, Gould t. 37 .

27. Syrniul, Sayg. Scotiaptex, Sro.

49. S. cinereus, Bp. S. cinerea, Gm. S. Eur. As. Am. s. barbata, Pall. S. lapponica, Sparrm.

Gould t. 42 .

*5o. S. Aluco, Boie, S. aluco et stridula, L. Europ. univers. Gould t. 47 .

As. occ.

28. Prxxx, Blight.

5r. P. onalense, Bl. S. uralens., Pall. $S$. Eur. or. s. et liturata, Retz. S. macrura, Natt. G. t. 44. Sibir.

\section{Subfamilia 21. Ululinae.}

29. Otus, Cup. Asio, Br. Agolius K. et Bl.

a. Otus, Ray.

*52. O. velgaris, Flem. Strix otus, L. Asio Eur. univ. As. otus, Strickl. Gould t. 39 . occ. Afr. s.

b. Brachyotus, Gould.

*53. O. вваспуотus, $S$. brach., Gm. S. aegolius, ulula, accipitrina, Pall. B. palustris, G. t. 40.

Eur. unir. As. Afr. Am. 
30. Ulela, Cuy.

54. U. nerulosa, Cuv. Strix nebulosa, L. Am.s. accid.Eura Gould. t. 46.

3I. Nrctale, Brehm, Scotophilus, Sw.

*55. N. funerea, Bp. S. tengmalmi, Gm. S. Eur. s. Sibiri funerea, L. S. passerina, Pall. G. t. 49 .

Subfamilia 22. Striginae.

32. Strix, L.

*56. S. FLAMME, L. Gould t. 36 .

Eur. As.

\section{ORDO III. PASSERES}

\section{Sertio 1. Ambulatores}

Familia 6. Caphimulgidae.

Subfamilia 24. Caprimulgincie.

33. Caphimelgus ${ }_{j} \mathbf{L}$.

*57. C. eunopaeus, L. C. punctatus, Meyer. Eur. el As. s. ez Hirundo caprimulgus, Pall. Gould. t. 5r. Afr.aest. migr.

58. C. nuficolis, Temm. Gould t. 52 . Hisp. m, ex Afr.

\section{Subfamilia 25. Scotornithinae.}

34. Scotoris , Sw.

59. S. climacurus, Sw. C. longicauda, Steph. C. climacteris, Vieill. Gall, t. 122.

Afr. acc. Gallia merid. 


\section{Familia 7. Cupselidae.}

\section{Subfamilia 26. Cypselinae.}

35. Cypseles, I11. Apus, Scop.

"6o. C. Apus, III. Hirundo apus, L. Micropus Eur. Sibir. m. murarius, Mey. Gould t. 53. f. I.

"6r. C. áelba, Ill. H. melba, Gm. H. alpina, Europ. m. Afric. Scop. Gould t. 53. f. 2.

As, occ.

Familia 8. Hirundinidae.

Subfamilia 27. Hirundininae.

36. Cinelidor, Boic.

'62. C. Urbica, Boie. H. urbica, L. H. la- Europa As. s. gopoda, Pall. Gould t. 57 .

Africa $s$.

37. Cotrue, Boie. Biblis, Less.

*63. C. ripania, Boie, H. riparia, L. G. t. 58. Eur. univ, As.s. *64. C. nupestris, Boie, $H$. rupestris et mon- Eur. m. As. occ. tana, Gm. Gould t. 56 . Afr. $s$.

38. Hindxdo, L. Cecropis, Boie.

"65. H. alpestris, Pall. $H$. capensis, Gm. $\boldsymbol{H}$. As. or. Afr. acc. daurica, Sav. H. rufula, Temm. G t. 55. Eur. m.

66. H. botssoneav, Temm.

Hispan. Graecia Afr. $s$.

${ }^{* 67 . ~ H . ~ n u s t i c a, ~ L . ~ H . ~ d o m e s t i c a ~ P a l l . ~ G . ~ t . ~ 54 . ~ E u r . ~ A s . ~ A f r . ~ O c . ~}$ 


\section{Familia 9. Coraciadidae. \\ Subfamilia 28. Oriolinae.}

39. Orioles, L.

*68. O. Galbula, L. Coracias galb. Br. G. I. 7I. Eur. As. Afr. s. Subfamilia 29. Coraciadinae.

40. Conacias, L.

${ }^{*}$ g. C. Garrola, L. Gould t. Go.

Europ. As. occ. Afr.

Familia 12. Alcedinidae.

Subfamilia 35. Alcedininae.

4I. Centle, Boie. Ispida, Sw.

*7o. C. nudrs, Boie. Alcedo rudis, L. G. t. 62. As. Afr. Eur. or.

42. Alcedo, L. Ispida, Auct.

*7I. A. Ispida, L. Gould t. 6 I.

Eur. As. Afr. s.

Familia i3. Meropidae.

Subfamilia 35. Meropinae.

43. Merops, L. Apiaster, Br.

*72. M. aptaster, L. Gould t. 59.

* 7 3. M. Aegretius, Forskh. nec Licht. $\boldsymbol{M}$. persica, Pall. $\boldsymbol{M}$. superciliosus, Lath. $\boldsymbol{M}$. savigny, Levaill. Bp Icon. I. Av. t. 2.
Eur.m. Afr.s.

As. Afr. occ. Eur. m. 
Famitia I4. Upupidae.

Subfamilia 37. Upupinae.

44. UPEPA, L.

"79. U. epops, L. Gould t. 238.

Eur. As. Afr. s.

Familia 20. Menuridae.

Subfamilia 5o. Troglodytinae.

45. Troglodytes, Vieill. Anorthura, Renn-

*75. T. europaeus, Cur. Motacilla troglody- Europ. univers. tes, L. Tr. regulus, Mey. Gould t. $\mathrm{x} 30 . \quad$ As. occ.

Familia 2i. Certhidae.

Subfamilia 51. Certhinae.

46. Certuin, $\mathbf{L}$.

*76. C. pamiliaris, L. C. scandulosa, Pall. Eur. univ. As. s. Gould t. 237 .

Subfamilia 52. Tichodrominae.

47. Tichodroma, Ill. Petrodroma, Fisill.

*77. T. muraria, Ill. Certhia muraria, L. Eur.m. As. occ. T. phaenicoptera, Temm. Gould t. 239. 
Subfamilia 56. Sittinae.

48. SITTA, L.

* 78. S. sтniaca, Ehrenb. S. rupestris, Cantr.

$S$. rufescens, $S$. neumareri, Hichab. $S$.

As. occ. Eur. m. saxatilis, Schinz, Gould t. 235 .

"79. S. europaea, L. S. caesia, Wolf el Mey. Eur. univers. Gould t. 234 .

8o. S. uralensis, Licht. S. eur. rar. sibirica, Pall. S. sericea, $S$. asiatica, Temm. G t. 236 .

As. s. accid. Europa or.

Familia 22. Paridae.

Subfamilia 57. Parinae.

49. LophopHanes, Kaup.

8r. L. eicolor, Bp. Parus bicolor, L. Gould t. 152 .

Am. s. acc. Scandin. nisi tantum Groenl.

*82. L. cristatus. Kaup. Parus cristalus, L. Gould t. 156.

Eur. s. et occ. acc. m. et or.

50. Parus, L.

*83. P. cranos, Pall. Gould t. 153.

As. s. hyem. Eur. bor. ad or.

*84. P. Caerderess ; L. Cyanistes caerulea, Kaup. Gould t. 154.

Eur univ.As.s. et or.

-85. P. мајов, L. Parus fringillago, Pall. Gould t. 150 .

Eur. univ. As. s. med. et or.

*86. P. Ater, L. Parus carbonarius, Pall. Gould t. r55. f. r.

Eur. As. etiam or. 
*87 P. Palustris, L. Paecile palustris, Kaup. Eur. et As. s. Gould t. 155. f. 2 .

*88. P. sibiricus, Gm. P. lugubris, Zetterst. As s. et Eur. or. Gould t. 151,f, 1. 2. etiam m.

51. Mecistura, Leach. Orites, Mroshe,

89. M. Cadodta, Leach. P. caudatus, L. La- Eur, m. As. s. nius biarmicus, L. Fn. S. Gould t. 157 et or.

52. Pavunus, Koch, Calamopliilus, Leaoh.

*9o, P. вiaricus, Koch, $\boldsymbol{P}$. biarmicus, L. Europ. univers. P. barbatus, Br. Gould t. $158, \quad$ As. $s$ et or.

53. Egithalus, Vig. Paroides, Koch.

"93. R. pendulines, Vig. P. pendulinus, L. Eur.m. et centr. Gould $t \quad 159$.

As. occ.

Familia 24. Alaudidae.

Subfamilia 62. Alaudinae.

54. Melayoconypha, Boie, Londra, Sykea.

92. II. tatarica, Boie. Al. tartarica, Pall. Tanagra sibirica, Sparrm. Saxilauda tartarica, Less. Gould t. 16 r.

*93. M. Calandra, Boie. Al. calandra, L. Eur.m. As.occ.

Londra calandra, Sykes. Gould t. 162. Afr. s.

55. Oroconis, Bp. Phylamnis, G. Gr.

"94. O. alpestris, L. Al. alpestris, L. Al. nivalis, Pall. Gould. t. 164 .

95. O. scriba, Bp. Phileremos scriba, Gould Proceed. Zool. Soc.
Eur. m. or. et As. finit.

Eur. et As, bor. acc. Italia. As. m. Eur. m. ad or. 
56. Calandella, Kaup.

96. C. leeucoptera, Bp. A. leucoptera, Pall. A. sibirica? $\mathrm{Gm}$.

${ }^{*} 7$. C. arenaria, Bp. A. calandrella, Bon. $A$. arenaria, Vieill. $A$. pispoletta, Pall. A. braclidactyla, Leisl. Gould t. 163. Al. kollyi, Temm. est monstr.

\section{Alauda, L.}

*98. A. cantarella, Bp.

Italia.

*99. A. Anvensis, L. A. caelipeta, Pall. G. t. 166.
Eur. or. As. s. et occ.

Eur. m. et As. occ.

58. Galerida, Boic. Lululla, Kaup.

*100. G. arbores, Boie Alauda arborea, L. Eur. As. occ. A. nemoralis, Gm. Gould t. 167 .

" Io . G. cristata, Boie Alauda cristala, L, Eur.med. et m. A. galerita, Pall Gould t. $\mathbf{1 6 5}$. As. Afr. s. 102. G. isabelina, Bp. A. deserti? Licht. Graecia, As. occ. A. isabellina, Temm. pl. col. 224. f. 2. Afr. or.

59. Centulladda, Sw. Alaemon, $\boldsymbol{K}$. et Bl. Corydalis, Temm.

*ro3. C. Doponti, Siv. A. duponti, Vieill. Fn. Fr. t. 36. f. 2. Roux Orn. Prov. 186.

*ro4. C. desertorua, Bp. A. desert. Stanl. A. bifasciata, Licht. G. t. $\mathbf{I} 68$.
As. occ. Afr. s. Eur. m.

Afr. s. Arcipel, acc. Sic. Hisp. Gall.m. 
Familia 25. Motacillidae.

\section{Subfamilia 63. Anthinae.}

60. Cormdalea, Vig.

* ${ }^{05}$. C. richardi, Vig. Antlius richardi, Vieill. A. rupestris, Mén. nec Auct. G. t. 135.

6!. Antuts, Bechst.

a. Agrodroma, Sw.

* rof. A. canipestris, Mey. Alauda campestris, Bechst. A. rufescens, Temm. G. t. 137 .

b. Anthus, Kaup. Spipola, Leach.

*io7. A. spinoletta, Bp. A. aquaticus, Bechst. Alau. spinoletta, L. Buffopl. enl. 66r. f。 2.

108. A. obscunus, K. et Bl. Al. obscura, Penn. A. petrosus, Anglor. A. rupestris, Nilss. A. littoralis, Br. A. aquaticus. Gould t. 138.

$$
\text { c. Leimoniptera, Kaup. }
$$

* Io9. A. Cervinus, K. et Bl. Mlot. cervina, Pall. A. pratensis, Erersm. A. cecilii, Aud. A. rufogularis, Brehm. G. t. 140 .

*iro. A. pratensis, Bechst. Al. pratensis, $\mathbf{L}$. Gould t. 136.

$$
\text { d. Pipastes, Kaup. }
$$

*iri. A. arboreds, Bechst. Motucilla spipola, Pall. Al. turdinae, Scop. G. t. 139 .
Eur. m. et oce, As. occ. Afr.

Eur.pr. or. As. occ. Afr. ad or.

Eur. m. et ceutr. As. occ.

Eur, s.

Eur, or. As. s. Afr. occ.

Eur. tota polarem usque.

Eur. As. Afr. s. 


\section{Subfamilia 64. Motacillinae.}

\section{Bupytes, Cuy.}

*ir B. flava, Cuv. Mot. flava et boarula, L. Eur. As. Afr. s. M. flava, Gesn. MI. flaveola, Pall. M. neglecta, Gould t. 146 .

*is 3. B. cinereocapilla, Bp. M. flava var. K. Eur. m. As. Afr. et Bl. $\boldsymbol{M}$. cinereoc. Savi. Bp. Icon. I.

*114. B. melanocephala, Licht. M. flava var. Eur. m. As. Afr. K. et Bl. MI. melan. Savi. Bp. Icon.

*155. B. Campestris, Bp. Mot. flava, Ray. MI. flaveola, Temm. nec Pall. Budytes rayi, As. s. et Eur, or. Gould t. 145 .

*116. B. crtreola, Bp. Mr. citreola, hinc citrinella, Pall. Gould t. 144. aest. Ins. Brit.

As. s. Rossia or. Europ. reliq. accid.

\section{Motacilla, $\mathbf{L}$}

a. Calobates, Kaup. nec Auct.

*it. M. sulpherea, Bechst. Mot. boarula,

Eur.m.et centr. Penn. nec. L. M. melanope, Pall, G. t. 147 . Ins. Brit. Afr. As. c. Oc?

\section{b. Motacilla, Cuv.}

"118. M. 4 т.вA, L. Mot. albeola, Pall. Gould t. 143 .

Eur. univ. As. Afr. occ.

*119. M. Yarnelit, Gould $M$. alba, var. Keys. et Bl. Gould t. 14 I.

Eur. s. pr. Ins. Brit. acc. It.

*120. MI. Lugubris, Temm. Mot. albeola, var. Pall. Gould t. 142.

Afr. or. acc. Furop. $u$. 


\title{
Familia 26. Turdidae.
}

\section{Subfamilia 65. Cinclinae.}

64. Crixclus, Bechst. Hydrobata, Vieill.

*121. C. AQuaticus, Bechst. Sturnus cinclus, L. Merula aquatica, Br. G. tab. 83 et 84 .

Eur. et As, s. oce.

Subfamilia 68. Ixodinae.

65. Ixos, Temm. Haematornis, Sw.

I22. I. ogscunus, Temm.

Andal. et Afr. s.

\section{Subfamilia 69. Turdinae}

\author{
66. Turdes, L. \\ a. MIerula, Ray.
}

*123. T. merula, L. Merula pulgaris, Ray. Eur.As.occ.Afr. Gould t. 72 .

S. Ins. Azor.

*124. T. torquatus, L. Merula torquata, Br. Europ. As. occ. Copsychus torquatus, Kaup. G. t. $73 . \quad$ h. As.Afr.occ. 125. T. sibiricus, Pall. T. leucocillus, Pall. As. s. Eur. or. IHerula sibirica, Bp. Gould t. 82. acc.

"26. T. atrigularis, Nalt. T. bechesteini, Naum. As. s. acc. Eur.

t. 6g. f. x. Cichloides bechsteini? Kaup. or. T. fuscatus ${ }^{\beta}$ Pall. Gould t. $7^{5}$. 
b. Turdus, Ray.

127. T. mgratorius, L. Gould t. 74.

Am.s.acc.Germ. et Brit.

*128. T. naumann, Temm. T. ruficollis? Pall. As. s. acc. Eur. T. dubiusi Bechst. Naum. Gould t. 79 . $\mathrm{m}$.

*r29. T. pilanis, L. T' musicus, Pall. Arceuthornis pilaris, Kaup, Gould t. $7^{6}$.

Eur. praes. s.As. occ.

* 3 o. T. viscrvonus, L. Ixocossyphus viscivo-

Eur. hyeme m. rus, Kaup, Gould t. $77^{\circ}$

${ }_{1}{ }_{1}$ 1. T. musicus, L. Turdus pilaris, Pallas, Gould t. 78 . f. I.

Eur. univ. As. Afr. or.

${ }^{\star}$ 132. T. iliacus, L. T. illas, Pall. G. t. 78 . f. 2.

Eur. univ. As. s.

* 33 . T. minon, Gm. Muscicapa guttata? Pall. Wils. Am. Orn. v.

Am. s. acc. ctiam Ital.

*34. T. rallidus, Lath. $T$. pallens, Pall. $T$. iliacus, var. Naum. $T$. seyffertitïi, Brehm. $\boldsymbol{T}$. werneri? Gené, Gould t. 80.

135. T. auroreds, Pall. T. bechsteinii, Naum. t. 69 . f. 2 .

Am. s. occ. As. or. acc. Eur.

67. Oreocincla, Gould.

136. O. AUREA, Bp. $\boldsymbol{T}$. aureus, Hollandr. $\boldsymbol{T}$. As. Oc. acc. Ger. whitei, Eyton. T. varius, Yarr. G. t. 8 I.

Brit.

\section{Subfamilia jo. Calamoherpinae.}

68. Caldhomerpe, Boie. Acrocephalus, Naum. quid?

${ }^{\star} 137$. C. Turdordes, Boie. Turdus arundinaEur.m. et c. As. ceus, L. T. junco, Pall. Sylvia turdoietiam or.Afr.s. des, Mey. Gould t. Io6.

138. C. olvetonu, Bp. Salicaria oliveto- Insul. Jon. rum, Strickland, Gould t. $10 \%$.

* 39 . C. arundinacea, Boie. Curruca arundi- Eur. m. et occ. nacea, Br. Gould t. iūos. 
*i4o. C. palustris, Boie, Sylvia palustris, Eur. c. As. occ. Bechst. Gould t. rog.

Italia s.

Í1. C. nignifnoxs, Bp. Sylv. nigrifrons, Boie. German. rariss.

69. Agrobates, Sw. Edden, Boie, nec Vieill.

*142. A. Galactodes, Bp. Sj-7v.galactodes, Te'nm. Turdus rubiginosus, Mey. Sylvia familiaris, Ménétr. Gould t. I12.

70. Cisticola, Less.

*143. C. schaenicola, Bp. S. cisticola, Temm. Eur. m. Afr. Gould t. I 3 .

7I. Calamodyta, Bp.

a. Calamodyta, Meyer.

*I44. C. melanopogon, Bp. Sylvia melanopogon, Temm. Lusciniola melanopogon, $\mathrm{Gr}$. S. bonellii, Brehm, Gould t. III. f. 2.

* 4 45. C. Aquatrca, Bp. S. schaenobaenus, Scop. Eur. occ. et m. S. aquatica, Lath. S. salicaria, Bechst. Gould t. III. f. 2.

* 46. C. phragmitrs, Bp. Mrot. schaenobae- Eur. As. Afric. nus, L. S. phragmitis, Bechst. G. t. rro.

147. C. Caricetr, Bp. Sylvia cariceti, Naum. tab. 82. fig. 2. 3 .

$$
\text { b. Locustella, Gould. }
$$

*if8. C. lanceolata, Bp. S. lanceolata, T'emm. Eur. med. accid.

*I49. C. locustella, Bp. S. locustella, Lath. Eur. As. s. Locusiella rayi, Gould t. 103. Eur. in.

As. Afr. accid.

Eur. m.

Eur. m. et or. or.

Eur. med. 
72. Cettia, Bp.

* 150 . C. Altisonans, Bp. Sylv. celti, Marmorá. Gould t. I 14 .

15ı. C. Sericea, Bp. Sylvia sericea, Nalter. Gould t. I 5 .
Eur.m.Ins.medit.

As. occ.

Eur. m. ad or. Afr. s.

73. Lescisiopsis, Bp. perdo-luscinia, Bp. 1834.

152. L. fluviatilis, Bp. S. Aluviatilis, Mey. Eur. or. rar. Locustella fluviatilis, Gould t. Ib2.

*153. L. savı, Bp. Sylvia luscinioides, Savi. Ital. Galloprov. S. savii, Vieill. Gould t. 104 . Russ, m. Afr.

74. Hrpolais, Brehm. Phyllopneuste, Gr. neo Auck.

*54. H. saltcania, Bp. Mot. hippolais, L. Europ. fere lula S. polyglotta, Vieill. Gould t. 133 . exclusa Brit.

b. var. Durazzi.

* 55 . H.? jcterina, Bp. Sylv. icterina, Vieill. Eur. m. et med. Gould t. 132 .

\section{Subfamilia 7 r. Sylvinae.}

75. Pitllopreuste, Mey. Phylloscopus, Boie.

*i56. P. simitatnix, Bp. S. sibilatrix, Bechst. Eur. m. et c. S. sylvicola, Lath. Gould t. I3r. f. 3.

*157. P. твосиrus, Bp. M.troclitlus, et acredula, L. S. fitis, Bechst. G. t. I3 г. fig. I.

Eur. etiam bor. As. Afr. or.

*158. P. Rufa, Bp. S. rufa, Lath. S. lippo- Eur. univers. lais, Angl. ex err. Penn. S. abietina, Nilss. Gould t. 13 I. f. 2 .

*159. P. воnelli, Bp. S. bonnelli, Vieill. $S$. Eur. m. accid. nattereri, Temm. S. prasinopyga, Licht. Gould t. I34.

Eur. med. 
76. Regulus, Ray.

"I6o. R. Proregulus, K. et Bl. Mot. proregulus, Pall. Regulus modestus, G. t. I49.

*i6r. R. cristatus, Ray. Mot. regulus, L. $\boldsymbol{R}$. flavicapillus, Naum. Gould t. 148. f. 2.

*162. R. Ignicaprlues, Cuv. S. ignicapilla, Br. R. pyrocephalus, Br. G. t. I48. f. 1 .

77. Mecizophilus, Leach.

*163. MI. provincialis, Leach. Mot. provincialis, Gm. S. dartfordiensis, Lath. G. t. 129.

78. Prroputhalma, Bp.

* 164 . P. melanocepuala, Bp. Mot. melanocephala, Gm. S. rusticola, Vieill. G.t. x23.

${ }_{1}$ 65. P. sarda, Bp. S. sarda, Marm. G.t. 127.

79. Srlvis, Lath.

a. Sterparola, Bp.

*166. S. conspicillata, Marm. Sterparola conspicillata, Bp. Gould. t. 126.

${ }^{*}{ }_{167}$. S. subalpina, Bonelli. S. leucopogon, Mey. S. passerina, Temw. S. mystacea, Mén. Gould t. 124.

\section{b. Sylvia, Bp.}

*168. S. curnuca, Lath. Curruca garrula, Br. Mot. Sylvia, Pall. Gould t. 125. f. 2.

${ }_{1}^{*} 69$. S. cinerea, Bp. Curruca cinerea, Br. Mot. sylvia, L. Gould t. 125. f. 1.
Eur. or. As.'s acc, etiam Br.

Eur. etiam bor. et or. As.

Eur.m. et occid.

Eur. m. et oce.

Ins. Britann.

Eur. m.

Sardin. Ital.

Italia m. Sard. Hisp. $m$.

Eur. m. As. et Afr.

Europ. etiam. b. As.m.ets. Afr. Europ. As. occ. Afr. or. 
${ }^{{ }_{2}}$ zo. C. hontensis, Penn. So hortensis, var.

Eur. univers。

b. Lath. S. salicaria? L. Gould t. I2r.

${ }^{*}$ 171. C. orphea, Boic. Curruca, Br. S. or- Eur. m. et c. plea, Temm. Gould t. Irg.

*i72. C. atricapilla, Br. Mot. atricapilla, L. Eur. etiam bor. Mot. moschita, Gm. S. atricapilla, Lath. Gould t. 120.

73. C. ruppelix, Bp. S. capistrata, Rupp. S. ruppelli, Temn. Gould t. 122 .

As. Afr.

Ins. or. Med. et II. rubr.

81. Adophoyeds, Kaup. Nisoria, Bp.

${ }^{*}$ I74. A. nisorres, Kaup, S. nisoria, Bechst. N. undata, Bp. Gould t. 128 .

Eur. centr. et or. As. occ.

82. Pullonela, Br. Daulias, Boie.

${ }^{*}$ 175. 1. mayor, Sw. Sylv. philomela, Bechst. Eur. s. et or. As. MI. aedon, Pall. Lusc, major, Br. G. t, II 7 . Afr. occ.

${ }^{*} 76$. P. uuscinia, Sw. Mot. luscinia? L. Eur. univ. As. S. luscinia, Lath. Luscinia plilomela, Bp. Lusciola luscinia, K. et Bl. G.t. 116 . occ. Afr. or.

83. Idena, K. et BI. Lusciola, K. et Bt.

177. I. caligata, Gr. Mot. salicaria, Pall. necL. Eur. or. As. s. S. caligata, Licht. $\boldsymbol{L}$. caligata, $\mathbf{K}$. et $\mathrm{Bl}$.

84. Accentor, Bechst. Prunella, Vieill.

${ }^{*}$ 178. A. alpinus, Bechst. 2Uot. alpina, Gm. Sturnus collaris, Scop. Gould t. 99 .

Mont. Eur. m. Eur. et As. s。

179. A. Montanelute, Temm. Mot. montanella, Pall. Gould t. гог.

As. s. acc. Eur. or. 
*180. A. modularis, Cuv. Mot. modularis, L. Eur. univ. As. Curruca sepiaria, Br. Gould t. 100.

occ.

\section{Subfamilia 72. Saxicolinae.}

85. Cisliope, Gould, Melodes, K. et Bl.

x8r. C. Camtschatrensis, Strickl. Mlot. calliope, Pall. Turdus kamtschatkensis, Gm. Accentor calliope, Temm. Calliope lathami, Gould t. In 8 .

86. Rubecula, Br. Dandalus, Boie.

*182. R. FamiLIARts, BI. MIot. nubecula, L. Dandalus rubecula, Boie. Gould t. $9^{8}$.

87. Craxecula, Br. Pandicilla, $\boldsymbol{B} l$.

${ }^{*}$ 183. C. suecica, Boie. Mot. suecica, L. Mot. caerulecula, Pall. So wolfii, Br. G. t. 97.

88. Roticills, Ray, Phaenicura, Sw.

*84. R. phaenicura, Bp. Mot.phaenicurus, L. Eur. As. Afr. Grould t. $9^{5}$.

*185. R. erithaca, Bp. Mot. erithaca, L. Sylv. tithys, Scopoli. Gould t. $9^{6}$.

186. R. Абловед, Bр. Mot. aurorea, Pall. Mot. erytlirogastra, Guldenstein.

89. Prativcola, Kaup, Saxicola Bechst.

${ }^{*}$ 87. P. rubicola, Kaup. Mot. rubicola, L. Eur. As. Afr. s. Sax. rubicola, Bechst. Gould t. 94 .

* 88. P. robetra, Kaup. Mot rubetra, L. Eur. Afr. Sax. rubetra, Bechst. Gould t. $9^{3}$.
As. s. ad or. acc. Eur. or.

Eur. unir. As. occ. Afr. or.

Eur. eliam bor. As. s. Ifr. s.

Eur. univ. As. Afr. s.

As. occ. et Eur. or. fin. 
90. SixicolA, Bechst. Vitiflora, Br. Oenanthe, Vieill.

* 8 g. S. oexanthe, Bechst. MTot. oenanthe, L. Vitiflora oenanthe, Boie. MI. vitiflora, Pall: Eur. univ. As. Gould t. $9^{0}$.

rgo. S. saltator, Men. Mot. strapazina, Pall. S. squalida, Eversm. Afr. s: Groenl. Eur. finit. ad or. As. occ. Afr. or.

*igr. S. stapazina, Koch. Vitiflora rufescens, Br. Eur. m. As. Afr. Oenanthe stapazina et albicollis, Vieill. Sax. aurita, Temm. Gould t. 91. 92.

192. S. Leucometa, K. et BI. Mlot. longirostris, Gm. Mlot. leucomela, Pall. minime vero Sax. leucomela, Temm. quae Sax. lugens, Licht. sp. Afr. Gould t. 89.

* $9^{3}$. S. Levednos, K. et Bl. Turdus leucurus, Gmel. Sax. cachinnans, Temminck; Gould t. 88 .

Eur. or. finit. As.

Eur. m. As. Afr. 91. Petrocincla, Vig. p. Monticola, Boie.

${ }^{*} 194$. P. saxatriss, Vig. Turdus saxatilis, L. T. infaustus, Latb. Gould t. 86.

Eur.m. et c. or. As. occ. Afr. s.

92. Petrocossmius, Boie.

"195. P. cyands, Boie, Turdus cyanus, L. T. so- Eur. m. Afr. 3. litarius, Hasselq. Gould t. 87 . 


\section{1}

\section{Familia 27. Muscicapidae.}

\section{Subfamilia 74. Muscicapinae.}

93. Butalis, Boie. Huscicapa, Sundev.

${ }^{*}$ r6. B. grisola, Boie, MIusc. grisola, L. G. t.65. Eur. univ. Afr.

94. Muscicapa, L. Butalis, Sundev.

*197. M. a tricapilla, L. Ember. luctuosa, Scop. Eur. eliam bor. Musc. luctuosa, Temm. Gould t. 63. f. I.

*198. Mi. Aj.bicollis, Temm. M. collaris, Bechst. Eur. m. et c. As. Musc. atricapilla, Jacq. Gould t.63.f.2. or. Afr. s.

95. Enfturosterina, Bp.

*199. E. paria, Bp. Muscicapa parva, Bechst. Europ. centr. et Gould t. 64 . or. acc. Ital.

Familia 28. Ampelididae.

Subfamilia 89. Ampelidinae.

96. Ampetis, L. Bombycilla, Bra

*200. A. Garkteles, L. Lanius garrulus, L. Eur.s. As. s.or. B. botemica, Br. Parus bombycilla, Pall. acc.num.Eur. Bombycivora poliocephala, Mey. G. t. I6o. centr. et m.

Familia 29. Lanidae.

Subfamilia 85. Laninae.

97. Laxius, $\mathbf{L}$.

a. Lanius, Boie. Collurio, rig.

201? L. мaJor, Pallo.

Eur.s.ad or.As.s. 
*202. L. excubitor, L. Collurio excubitor, Vig. Eur. etiam bor. Gould t. 66.

"203. L. meridionalis, Temm. Collurio meri- Eur. m. ad occ. dionalis, Vig. Gould t. 67 . Afr. s. acc. It.

b. Enneoctonus, Boie, Collurio, Br.

*2o\%. L. minor, Gm. L. italicus, Lath. L. vi- Eur. m. et med. gil, Pall. Gould t. $68 . \quad$ As. occ.

* 205. L. collurro, L. L. aeruginostes, K1. Eur. etiam bor. L. spinitorques, Bechst. Gould t. 69. As. Afr.

*206. L. Rufus, Br. L. ruficeps, Bechst. L. $\mathrm{ca}$ - Eur. m. med. et staneus, Risso? Gould t. jo. occ. Afr. s.

98. Telophords, Sw. Laniarius, Boie nec Vieill.

207. T. senegalus, Bp. L. senegalus, L. L.ery- Eur. m. ad or. thropterus, Shaw. L. cucullatus, Temm. Afr.

\section{Famitia 3o. Corvidae.}

\section{Subfamilia 88. Garrulinae}

99. Perisoreds, Bp. Dysomittia, Sw.

208. P. infaustus, Bp. Corvus infaustus, L. Eur. et As. pol. C. mimus, Pall. Lanius infaustus, L. acc. Eur. med. Gould t. 2 r5.

I00. Ganntles, Br.

* 209. G. Glandarius, Br. Corvus glandarius, L. Eur. univ. As. s. Garrulus pictus, Koch. Gould t. 215.

гіо. G. atricapilles, Is. G. Garrulus me- As. occ. et finit. lanocephalus, Gené, t. in Act. Taur.

Eur. or.

$$
\text { I0I. Pica, Br. }
$$

21. P. cyanga, Wagl. Corvus cyanus, Pall. Hispan. As. m. Gould t. 2 r 7 . et or. 


\title{
43
}

*212. P. Gaddata, Ray, C. pica, L. P. vulgaris, Hempr. P. melanoleuca, Vieill. $\boldsymbol{P}$. albi-

Eur. univ. As. s. ventris, Vieill. $\boldsymbol{P}$. europaea, Cuv. G.t. 216. etiam or.

\section{Subfamilia 9o. Corvinae.}

\author{
102. Coryus, L.
}

a. Mronedula, Brm.

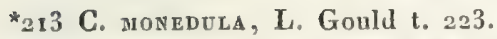

Eur. univ. As. S.

294. C. SPERmologus, Vieill. Monedula tur-

Gallia Germ. rium, Brm. Frisch Vog. Deutschl. t. 68.

b. Corvus, Brm.

*215. C. connix, L. Gould t. 222.

Eur.As.s. ad occ.

*216. C. Corone, Lath. nec L. Gould t. 221.

Eur.As.etiamor.

$\star_{2 \text { r }}$. C. conax, L. Corvus maximus, Scop. Gould t. 220.

Eur. As.s. etiam ad or.

218? C. Leucophaeds, Vicill. Corvus borealis albus, Br. Vieill. Gal. Ois, t. 100.

Ins. Ferroe, Eur. et As. bor.

*219. C. frugitegus, L. C. corone, L. G. t. 224. Fur. et As. s.

103. Fregilus, Cuy.

*220. F. Graculus, Cup. C. graculus, et eremita, L. Fr. erythroramplus, Dum. Gould t. 2 rg.

Alp. Eur. Ins.

Br. As. s.

104. Pyrmocorax, Cuv.

*221. P. alfinus, Vieill. Corvus pyrrhocorax, L. Gould t. 218.

Alp. Eur. m. et As, occ. 
105. Nucifnaga, Br.

*232. N. garyocatactes, Br. C. caryocalactes, Sept. el mont.

L. Caryocatactes guttatus, Nilss. G.t.213. Eur. As.

\section{Subfamilia 93. Sturninae.}

106. Stunves, $\mathbf{L}$.

*223. S. vulgaris, L. St. varius, Wolf, G.t. 2 ro. Eur. As. Afr. s.

*224. S. unicolor, Marra. Gould t. 211 . E.m.praes. Ins.

107. Acridotueres, Ranz. Pastor, Temm. Boscis, Bm.

*225. A. noseus, Ranz. Turdus roseus, L. IIerula rosea, Br. Pastor roseus, Temm. Gracula rosea, Glog. Psaroides roseus, As. Afr. Eur. or. irregular. occ. Vieill. Boscis rosea, Brm. Pecuarius roel in. seus, Temm. Thremapliilus roseus, Macgill. Nomadites roseus, Peteniz, Gould t. 212.

Familia 3 i. Fringidlidde.

Subfamilia 98. Emberizinae.

108. Cxpcunauus, Bp. Mtiliaria, Brm.

*226. C. mriarra, Bp. Emberiza miliaria, L. Eur. fere univ. M. europaea, Sw. Grould t. I $7 \mathbf{r}$ As. oce.

109. Euberiza, L.

a. Emberiza.

*227. E. citrinelia, L. Gould t. 173.

Eur. praeser. s.

*228. E. aureola, Pall. Fringilla pinetorum, Lepech. Gould t. 174 .

As. s. praes. or. acc. Eur. or. 
*229. E. cirlus, L. Gould t. 175 .

*33o. E. hortulanus, L. Gould t. 176.

*23r. E. Caesra, Gretzschm. Gould t. i8r.

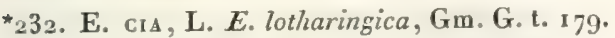

233. E. pithyornus, Pall. Fringilla dalmati$c a$ ? Gin. Gould t. 180 .

234. E. curysophrys, Pall. Selys Faun. Belg. t. 4 .

235. E. Bonapartir, Barthelemy de la Pomm.

236. E. cinerea, Strickl. Nm. (a).

$\star_{23} 3$. E. nustica, Pallas, Emb, lesbia, Calvi, E. borealis, Zett. Gould t. 177 .

238. E. fucata, Pall. Emb. lesbia, Temm. nec Gm? Gould t. 178 .

239. E. striolata, Licht.

*240. E. durazzi, Bp. Emb. provincialis? Gm. Ic. Faun. It. Av. t. 6.

\section{b. Schoeniclus.}

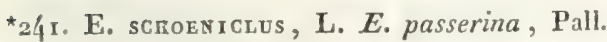
Gould t. 183 .

*2\{2. E. Pynhulordes, Pall. E. palustris, S. Emb. caspia, Ménétr. Gould t. i82.

I10. Plectrophaves, Mey.

*243. P. mivalis, Mey. E. nivalis, L. E. montana et mustelina, Gm. Gould t. 170 .

*244. P. Lapponicus, Selby. Fringilla lapponica, L. Fr. calcarata, Pall. Emb. calcarata, Temm. Centrophanes calcaratus, Kraup. Gould to 169 .
Eur. As. occ.

Eur. As. occ.

As.Afr. acc. Eur.

Eur. As. oce.

As. s. ad occ.

Eur. or.

As. acc. Eur.

Acc. Gall. $\mathrm{m}$.

As. s. Eur. or.

As, s. ad or. Eur.

bor. acc. $m$.

As.s. ad or. acc.

Eur.

Hispan. Afr. s.

Ital. occ. Gallia

m.

Eur. univ. As. S.

Eur. m. et or. As. occ.

Boreal. utriusq. cont.h.acc.m.

Boreal. utriusq. cont. hyeme acc. $m$.

(a) E. hyperborea, pusilla, spodocephala, rutila, Pall. sunt species Asiaticae forsan in Europa orientali accidentaliter occurrentes. 


\title{
Subfamilia 99. Fringillinae.
}

\author{
III. EuspizA, Bp.
}

*245. E. Metanoceprata, Bp. Emb. melanoEur.or. As, occ. ceplaala, Scop. Tanagra melanictera, Guldenst. Xanthornus caucasicus, Pall. Fringilla crocea, Vieill. Emberiza granativora, Ménétr. Gould t. I 72.

I12. SthutuUs, Boie. p. Niphaea, Audub.

246. S. Hyemalis, Bp. Emberiza hyemalis, L. Emb. nivalis, Wils. Fr. Iudsonia, Forst. Gould t. Igo.

Am. s. acc. Eur. bor.

II3. Montifingilla, Br. Orites, $\boldsymbol{K}$. et $\mathrm{Bl}$.

*247. M. Nivalis, Br. Fringilla nivalis, L. Fr. saxatilis, Koch. Plectrophanes frinAlp. Eur. Caugilloides, Boie. Passer alpicola, Pall. cas. et Ceraun. Gould t. 189 .

II4. Frivgilla, L. Struthus, Boie.

*248. F. montifringilia, L. Fr. lulensis, L. Passer montifringilla, Pall. G. t. 188.

Eur. As.s. etiam or.

*249. F. carlebs, L. Passer spiza, Pall. FrinEur. As.s.et occ. gilla sylvia, Scop. Fr. spiza, Renn. Fr. nobilis, Schrank, Gould t. 187 . Afr. bor. Ins. Madeir.

I 5 . Coccoturaustes, Br.

*25o. C. vulganis, Ray. Loxia coccothraustes, L.

Eur. univ. As. s. C. europueus, Selby. C. deformis, Koch, eliam or. Gould t. 199. 
116. Prrgita, Cuy. Passer, Br.

*25r. P. salicaria, Bp. Fr. salicaria, Vieill. Ins. medit. Afr. Fr. hispaniolensis, Temm. Fr. sardoa, s. As. etiam or. Savi, Passer salicarius, K. G. t. 185. f. I. Ocean.

*252. P. italiae, Bp. Fr. italiae, Vieill. FrinItal. Carinthia. gilla cisalpina, Temm. Passer italicus, $\mathbf{K}$. et Bl. Gould t. I85. f. 2.

*253. P. donestica, Cuv. Fr. domestica, L. Passer domesticus, Gesn. G. t. 184.f. 1. Eur. (vix Ital.) As. Afr. s.

*254. P. montana, Cuv. Fr.montana, L. Passer montanina, Pall. Fr. campestris, Schr. Loxia hamburgia, Gm. G. t. 184. f. 2.

Europ. As. s. etiam or.

I17. Petroxu, Bp. Pyrgita, Ko et Bl.

*255. P. sтulta, Kaup. Fringilla petronia L. Fr. stulta, Gm. Petronia rupestris, Bp. Passer petronia, Koch, Gould t. 186.

Eur. m. Ins. Teneriff. As. occ. acc. Eur. s.

118. Cillonospiza, Bp. Ligurinus, Kocho nec Aut.

*256. C. incerta, Bp. Fringilla incerta, Roux. Eur. m. Ic. Faun. It. Av. tab. 8.

${ }^{\star} 25$ 7. C. Chlor1s, Bp. Loxia chloris, L. Frin- Eur. As. s. etiam gilla chloris, Mey. Coccothraustes chloris, Pall. Serinus chloris, Boie, G. t. 200. or.

I19. Serivus, Bp. Dryospiza, Ko. et Bl.

*258. S. meridionalis, Brm. Fr. serinus, L. Eur. m. Afr. s. S. Urumalis, Strickl. S. Iortulanus, Koch. acc. Eur. med.

S. flavescens, Gould t. $19^{5}$.

${ }^{\star} 25$ g. S. islandicus, Bp. Fr. islandica, Fab. Islandia.

Loxia serinus, Prodr. Orn. Isl. 
120. Citrinella, Bp. Dryospiza, Gro nec K. ot Bl.

*260. C. alpina, Bp. Fr. citrinella, L. Chl. citrinella, K. et Bl. Fring. brumalis, Eur.m.acc.Eur. Bechst. Fr. alpina, Scop. Serinus citrinella, Boie, Dryospiza citrinella, Gr. Gould t. $19^{8}$.

121. Conrysomirnis, Boie. Spinus, Brm., Ligurinus, $B r$.

*261. C. spinus, Boie, Fringilla spinus, L. Spinus viridis, Koch. Gould t. 197.

Eur. As. s. etiam or.

122. Cardeglis, Br. Acanthis, $\boldsymbol{K}$. et $\mathbf{B l}$.

*262. C. elfigans, Steph. Fring. carduelis, L. Eur. As. occ. C. nobilis, auratus, vulgaris, communis, Angl. Gould t. $19^{6}$.

$$
\begin{aligned}
& \text { 123. Lingta, Bp. } \\
& \text { a. Linaria, Br. }
\end{aligned}
$$

*263. L. linaria, Bp. Linaria rubra, Gesn. Bor.utrius. hem.

L. rufescens, Vicill. Fr. rufescens, Savi. hyem. E. m. Gould t. 194 .

264. L. вовеalis, Bp. Fr. linaria, Savi. Eur. so et occ. Fr. borealis, Vieill. Linaria canescens, $\Lambda$ s.s. etiamor. Gould t. $19^{3}$. Am. s.

\section{b. Cannabina, Brm.}

265. L. Brevinostris, Gould Proc. Zool. Soc.

"266. L. Flavinostris, Bp. Fringilla flavirostris, L. Fr. montium, Gm. G. t. $19^{2}$.

${ }^{*} 67$. L. cannabina, Bp. Fr. cannabina, L. C. linota, Gr. Passer papaverina, Pallas. Gould t. rgr.

Eur.finit.As.occ.

Europ. s. hyem. etiam m.

Europ. As. occ. Afr. or. 
124. EngtunospizA, Bp. Hamorrhous, Sw. Carpodacut p. Kaup.

268. E. pha enicoptera, Bp. Linaria phaenicoptera, Gould.

${ }_{2} 69$. E. githaginea, Bp. Fr. githaginea, Licht. Pyrr. githaginea, Temm. Gould t. 208.

27o. E. rosea, Bp. Passer roseus, Pall. Fringilla rosea, Pall. Carpodacus roseus, Kaup. Pyrrhula rosea, Temm. Gould t. 207.

${ }^{2}$ 27. E. eвтturina, Bp. Loxia erythina, Pall. Fr. erythrina, Mey. Pyrr. erytlirina, Temm. Fr. rosea, Lath. Loxia cardinalis, Beseke. Corythus erythrinus, K. et Bl. Erytlirotloorax rubrifrons, Brm. Gould t. 206.
Eur. finitim. As. occ.

Afric. er. accid. Eur. m.

As. s. ad or. acc. Eur. or.

Eur. or. As, s. acc. etiam in Eur. m.

125. UraGes, K. et B1. Carpodaçus p. Kaup.

272. U. sibiricos, K. et Bl. Pyrr. longicauda, Temm. Lox. sibirica, Pall. Pyrr. caltdata, Pall. Cor. sibiricus, Bp. Pyrr. sibirica, K. et Bl. Gould t. 205.

As. s. ad or. ace.

Fur. or.

\section{Subfamilia 100. Loxinae.}

126. Prrnuula, Br.

${ }_{27} 7$ 3. P. volgaris, Br. Pyrr. rubicilla, Pall. Lox. pyrrlula, L. P. europea, Vieill. Eur. As. s, etiam Lox. atra, Brunn. P. rufa, Koch. Emb. coccinea, Sarder. G. t. 209.

127. Conrtuus, Cuy. Strobilophaga, Fisili.

274. C. вubicilta, Bp. Loxia rubicilla, Guldenst. Coccothraustes caucasicus, Pall. Pyrrhula calcasica, K, el Bl.
As, occ. et finit.

Eur. or. 
*275̆. C. enucleator, Cuv. L. enucleator, L. Bor. Europ. As. L. psittacea, Pall. Fr. enucleator, Hey. Am.s.acc.Eur. $P$. enucleator, Temm. G. t. 204 . m. med.

128. LoxIA , Br. Crupirostra, Cury.

*276. L. pytropsittacos, Bechst. L. curvirostra, Otto. Cr. pinetorum, Mey. $\boldsymbol{L}$. curv. major, Gm. Gould t. 201.

*277. L. Convirostra, L. L. crucirostra, Pall. L. vulgaris, Ranz. Cr. inlgaris, Daud. C. abietina, Mey. Gould t. 202.

278. L. taentoptera, Glog. Cr. bifasciata, Brm. G. t. 203.

Eur. s. et med. acc. It. s.

Eur.univ. praes. s. As. s. ad or.

Eur.s. As.s. acc. Eur. med.

\section{Tribus 2. Scousours.}

Familia 35. Cuculidae.

Subfamilia ıо. Cuculinae. 129. Oxylopuos, Sw. Coccystes, Glog.

*279. O. Grandarius, Bp. Cuculus glandarius, L. C. pisanus, Gm. C. macrurus, Brm. Grould t. $24 \mathrm{r}$.

\section{Cuculus, $\mathbf{L}$.}

*280. C. canones, L. C. rufus, Bechst. C. borealis, Pall. C. cantor, III. C. cinereus, Besek. C. hepaticus, Sart. G. t. 240.

13I. Gureus, Boie. Piaya, Lese.

281? C. Americanus, Bp. Cuculus americanus, L. Coccyzus americanus, Vieill. Erytrophrys americanus, Sw. Gould t. 242. Eur. As. Afr. s.

As. Afr. Hisp. m. acc.It.ets.nidif.

Amer. s. accid. Eur. s. 
Familia 36. Picidae.

Subfamilia 111. Yunginae.

132. YuNx, L.

282. Y. товquilla, L. Picus yinx, Pallas. Europ. As. s. ex Gould t. 233 .

Africa.

\section{Subfamilia 113. Picinae.}

I33. Gecivus, Boie. Braohylophus, Sw.

*283. G. viridrs, Boie. Picus viridis, L. G. t. 226.

Eur. As. occ.

284. G. canus, Boie. P. canus, Gm. P. chloris, Pall. $\boldsymbol{P}$. norvegicus, Lath. P. cani-

Eur. med. et or. ceps, Nilss. $\boldsymbol{P}$. viridi-canus, Mey. G. t. 227.

As. s.

134. Drrocopes, Boie. Dryotomus, Sw.

*285. D. mantius, Boie. Picus martius, L. Eur. As. s. Grould t. 225 .

135. Picus, L. Dryobates, Boie. Dendroeopus, Koch.

*286. P. Levconotus, Bechst. P. cirris, Pall. Europ. s. et or. Dendrodromas leuconotus, líaup. Gould As. s. tab. 228.

*287. P. major, L. Picus cissa, Pall. G. t. 229. Eur. As. s.

*288. P. xiedius, L. P. cynaedus, Pall. G. t. 23o. Eur.

*289. P. arinor, L. P. pipra, Pall. G. t. 23 r. Eur. As. s.

136. Apternus, Sw. Picoides, Lacép.

*290. A. tridactrues, Sw. P. tridactylus, L. Eur. s. mont. Dendrocopus tridactylus, Koch. G. t. 232. Eur.med.As,s. 


\title{
ORDO IV. COLUMBAE.
}

\section{Familia 42. Columbidae.}

\section{Subfamilia 122. Columbinae.}

\author{
137. Coldmea, L.
}

*291. C. palumbes, L. Gould t. 243.

Eur, etiam s. As. s. hyem. Afr. s.

*292. C. aenas, L. Fn. Suec. Gould t. 244. Eur. etiams. As. occ. Afr. s.

*293. C. urvia; Auct. Columba aenas, L. Columba domestica, Gm. Gould t. 245 .

Eur. etiams. Afr. s. As. s.

I38. Tuntur, Ray. Peristera, Boie

294. T. senegatensis, Bp. C. senegalensis, $\mathbf{L}$. C. cambayensis et aegyptiaca, Lath. C. senegalensis et cambayensis, Temm. $C$. testaceo-incarnata, Forsk. C. maculicollis, Wagl.

*2g5. T. auritus, Ray. C. turtur, L. Per. Europ. etiam s. turtur, Boie. Tur. migratorius, Selb. As. s. Gould t. 246 .

296. T. nisorius, Selby. Columba risoria, L. Thrac. As. et Af.

139. Ecropistes, Sw.

297. E. migratories, Sw. Columba migratoria et canadensis, L. Wils. Am. Orn. t. 44 . f. $\mathrm{x}$.

Am. 3. acc. in Ins. Brit. 


\section{SUBCLASEIS II. GRALLATORES}

\section{ORDO V. GALLINAE.}

Familia 43. Pteroclidae.

Subfamilia 125. Pteroclinae.

140. Ptenocles, Temm. Oenas, Vieill.

*298. P. Alсuата, Licht. T. alchata, L. T. Eur. m. As, occ. caudaculus, Gm. T. cliata, Pall. Pt. Afr. $s$. setarius, Temm. $\boldsymbol{P}_{t}$. caspius, Mén. G. t. 258 .

*299. P. arenarius, Temm. T. arenarius, Pall. Eur.m. etiam ad Perdix aragonica, Lath. Oenas arena- occ. Afr. As. rius, Vieill. Gould 257 .

occ.

Familia 47. Phasianidae.

Subfamilia 133. Phasianinae

I41. Puasiand, L.

\#300. P. concruces, L. Gould t. 247 .

As. occ, et finit. Eur. Corsic.

Familia 48. Tetraonidae.

Subfamilia I34. Tetraoninae.

142. LAgopts, Vieill.

a. Lagopus, Kaup.

3oz. L. bracinactylus, Gould. Tetrao brachidactylus, Temm. Gould t. 256.

Eur. or. et As. max. bor. 
*3o2. L. moros, Leach. T. lagopus var. minor alpina, L. L. alpina, Nilss. T. rupestris, Gm. L. vulgaris, Vieill. L. rupestris, Boie. G. t. 253. 254?

3o3. L. albus, Bp. T. lagopus, L. T. cachinnans? Retz. T. albus, Gm. $T$. saliceti, Temm. L. saliceti, Sw. L. subalpina, Nilss. Gould t. 255.

$$
\text { b. Oreias, Kaup. }
$$

3o4. L. scotrcus, Vieill. Tetrao scoticus, Lath. Bonasa scotica, Br. Gould. t. 252.

$$
\text { 143. Tetrao, L. }
$$

a. Tetrao, Kaup. Lyrurus, Sw.

"3o5. T. tetrix, L. Tetrao betulinus? Scop. Gould t. 250 .

Eur. et As. s, et mont. acc.Ap.

Hybridus cum seq. T. urogallides, Nilss. T. medius, Leisl. T. hybridus, Sparrm. T. intermedius, Langsd. G. t. 249 .

Hybridus cum Lagopodis albi foem. T. LAGopides, Nilss. T. tetrix, mas, var. Sparr. cum. fig.

b. Urogallus, Kaup, Tetrao, Sw.

*3o6. T. unogalius, L. Grygallus, Gesn. Tetrao eremita? Thunb. Gould t. 248.

E. et As. s. mont. acc. Alp. m.

144. Bonasia, Bp. Tetrastes, Ko. et $\mathrm{Bl}$.

*307. B. sylvestris, Brm. T. Bonasia, L. Tetrao canus? Sparrm. B.betulina, Strickl. B. europaea, Gould t. $25 \mathrm{r}$.

Eur. s. et med. As. occ. 


\section{Subfamilia 135. Perdicinae.}

145. Fraxcolines, Steph. Attagen, $\bar{K}$. et $B l$.

\#308. F. vulgaris, Steph. T. francolinus, L. Sic.Melit.Arcip. Chaetopus francolinus? Sw. G. t. 259? Afr. As.

I46. Perdix, Br. Caccabis, Kaup, Cheeura, Hodgs.

*3og. P. Graeca Br. P. saxatilis, Bechst. P. rufa, S. G. Gm. Tetrao rufa, Pallas. Chacura graeca, Gr. Gould t. 26r. fig. 2.

*3ro. P. nuвra, Br. Perdix rufa, Gesn. Tetrao rufus, L. Gould t. 260.

*3i 1. P. petrosa, Lath. Alectoris petrosa, Kaup. Gould t. 26r f. I.

147. Staria, Bp. Perdix, Gr.

*3r2. S. pendix, Bp. $\boldsymbol{T}$. perdix, L. P. damascena, Lath. $\boldsymbol{P}$. cinerea, Br. $\boldsymbol{P}$. montana, Lath. Gould t. 262 .

I48. OrTrx, Steph. Colinus, Less.

3r3. O. virginiana, Bp. T. virginianus et marilandicus, L. $P$. borealis, Temm. O.borealis, Steph. Aud. Am. t. $7^{6}$.
As. occ. Gr.It.m. et occ. Cors. Gall.m. Helr. Il.,Gall.m.Hisp. Ins. Jersey et Guernes.

Misp.Ins Balear. Sard. Afr. s.

Europ. As. occ. Afr. s.

149. Coturvix, Bonn. Oriygion, $K$. et $B l$.

*314. G. comnunss, Bonn. T. coturnix, L. $C$. major, Br. P. colurnix, Lalh. C. dactylisonans, Mey. C. europaeus, Sw. C. vulAm. s. unde in Eur. Angl. garis, Jard. Gould. t. 268. 
Subfamilia 136. Turnicinae.

I50. Tunvix, Bonnat, Ortygis, $m$.

*315. T. Gibraltanicus, Bonn. Tetrao andalu-

Hisp. Sic. Afr. s. sica et gibraltarica, $\mathbf{G m}$. Hemipodius taacc. Gallia m. chydromus et lunatus, Temm. G. t. 264 .

\title{
ORDO VII. GRALLAE.
}

\section{Tribus 1. Gunlinarene.}

Familia 53. Charadridae.

Subfamilia I41. Otidinae.

\author{
I5I. OTIS, L. \\ a. Otis, Leach.
}

*316. O. TARDA, L. Gould t. 267 .

Eus. p. or. As.

b. Tetrax, Leach.

*31 7. O. тетвах, L. Tetrax campestris, Leach. Eur. Sard. Sic. Gould t. 269 .

As. occ. Afr. s.

c. Houbara, Bp. Chlamydotis, Less.

3ı8. O. moubara, Gm. Chlamydotis houbara, Less. O. macqueeni, G. O. Gr. Hou-

Afr. s. As. occ. bara undulata, G. R. Gr. Gould t. 268. ad m.acc. Eur.

\section{Subfamilia 142. Charadrinae.}

152. Edicremes, Temm.

*3rg. A. creprtans, Temm. Charadrius aedienemus, L. Gould t. 288.

Eur. etiam.s.As. occ. Afr. s. 
153. Corsonics, Lath. Tachydromes, $I h$.

"33o. C. Gallices, Bp. Charadrius corrira, Bom.

Ch. gallicus, Gm. Curs. europaeus, Lath.

Afr. 6. As. occ.

C. isabellinus, Mey. Grould t. 266.

acc. Eur. m.

154. Glareola, Br. Trachelia, Scop.

*3ai. G. pratincola, Pall. Hirundo pratincola, L. Gl. alstriaca, naevia et senegalensis, Gm. Gl. torquata Mey. G. t. 265.

Eur.praes.or.As. occ. Afres. et occ.

323. G. nordmanni, Fisch. G. pratincola, Pall. Eur, or. As. occ.

155. Cimaradrius, L. Egialites, Boie, Hiaticula, Mochr.

*333. C. mitrcula, L. Egialites hiaticula, Boie, Hiaticula annulata, Gr. Gould t. 296.

Eur. As. etiam or. Afr. s.

\#324. C. cunonicus, Beseke. Ch. liaticula, Pall. Eur. As. s. Ch. fluviatilis, Bechst. Ch. minor, Mey. Ch. intermedius, Ménétr. Gould t. 297.

*325. C. cantianus, Lath. Ch. alexandrinus, Eur. As. Hasselq. Ch. littoralis, Bechst. Cl\%. albifrons, Mey. Gould t. $29^{8}$.

326. C. руглнотновах, Temm. Ch.jugularis? As. occ. Eur. or. Wagl. Gould t. 299. ad $\mathrm{m}$.

156. Ecdromas, Boic. Morinellus, Gisn.

*327. E. morinelles, Boie, Ch. morinellus, L. Eur. praesertim Ch. tataricus, Pall. Gould t. 295 .

or. hyem. $\mathbf{m}$.

3.28. E. asiaticus, $\boldsymbol{K}$. et BI. Cl. asiaticus et As. occ. et finit. caspius, Pall.

Eur. or.

I57. Pluvialis, Br. Charadrius, Auct.

4329. P. apricarius, Bp. Ch. pluvialis el apri-

Eur.As.Afr.aest。 carius, L. Pluvialis aurea, Br. G. t. 294 .

bor. hyem. $m$. 
I58. SQuatarola, Cur.

*33o. S. helvetica, Cuv. Tringa helvetica, L. Boreal. hyem. Vanellus helveticus, Br. Tringa squata- per unir.Eur. rola, Gm. Gould t. 290.

\section{Subfamilia $\mathrm{x}$ 43. Vanellinae.}

I59. IIoploptends, Bp. philomachus, Gr.

33r. H. sprroses, Bp. Charad. spinosus, L. As.calid.Afr.Acc. Gould $t, 29^{3}$. Eur. or. ad m.

160. Cinetrusia, Bp.

*332. C. gregaria, Bp. Ch. gregarius, Pall.

As.occ. et extreTr. keptuschka, Lepech. T. fasciata, Gm.

C. wagleri, Gray. Gould t. 29 2. mit.or.Eur.m. acc. Ital.

I6I. Vanellus, Br. Gavia, Elein。

*333. V. cristatus, Mey. Tringa vanellus, L. Eur univ. Afr. V. Gavia, Steph. Gould t. 29r. As. occ.

\section{Subfamilia 144. Haematopodinae.}

I62. Strepsilas, 111. Ginclus, MLehy.

*334. S. intenpres, Ill. Tringa interpres, L. Boreal. hyem. Charadrius cinclus, Pall. Str. collaris, Eur. m. Temm. C.morinellus, Gr. G.t. 3 18.

163. Hienutopus, L. Ostralega, br.

*335. H. ostralegus, L. M. hypolelca, Pall. Eur. As. s. ad Gould t. 300 . occ. hyem. $m$. 


\title{
Familia 54. Scoloracidae.
}

\section{Subfamilia 145. Scolopacinae.}

\author{
I64. Scolopax, L. Rusticola, Mochr.
}

*336. S. rosticola, L. Rusticola 'ulgaris, Vicill. Eur. et As.unir. Gould t. 3rg. Afr. s.

165. Exalıus, Kaup, Tylocota, Ep. Homoptilura; Gr.

337 , E. sabrnif, Kaup. Scolopax sabinii, Vig. Acc.Ins.Britann. Gould t. 32I. f. I. unde?

I66. Gallivago, Steph. Telmatias, Boie, Ascolopax, $\boldsymbol{K}$. ot Bl.

a. Gallinago, Brm. Numenius, Gr.

*338. G. majon, Bp. Sc. major, Sav. Sc. media, Fr. Sc. paludosa, Retz. Sc. paluEur. praes. or. stris, Pall. G. 1. 320.

339? G. montaget, Bp. Sc. major, Mont. Eur. s. et med. *340. G. вкеимі, Bp. Sc. brehmi, Kaup. Pem Eur. med. et m: lorychius brehmi, Kaup. Ic. Faun. It. I.

*34 ז. G. scolopacinus, Bp. Sc. gallinago, L. Eur. As. s. Gould t. 32r. f. 2 .

b. Lymnocryptes, Kaup. Philolymnus, Br.

\#342. G. Gallinula, Bp. Sc. gallinula, L. Eur. As. s. Gallinago minima, Ray. Gould t. 322.

167. Macroramiplús, Leach. Lymnodromus, Wiedo

343. II. Griseus, Leach. Sc. grisea et novebo- Ar. s. acc.Eur.s: racensis, Gm. Sc. paykulli, Nilss. G.t.323. 


\section{Subfamilia 146. Tringinae.}

I68. Limicolı, Koch. Falcincllus, Kaup.

*344, L. PYgaAea, Koch. Num.pygmaeus, Lath.

Eur.or.et m. $\Lambda$ s。

N. pusillus, Bechst. Tr. platyrlyync-

ha, Temm. Tr. pygmaea, Sav. L. pla-

tyrliyncha, Gr. Gould t. 33 r.

I69. Pelidna, Cur. Schaenichus, Moekr.

a. Actodromas, Kaup.

*345. P. mavota, Cuv. Tringa minuta, Leisl.

T. cinclus, Pall. T. pusilla, Mey. G. t. 332.

Boreal. orb. ant.

b. Leimonites, Kaup.

*346. P. memmest, Cur. Tr. pusilla, Leisl.

Tr. temmincki, Leisl. Gould. t. 333.

Afr.

$$
\text { c. Pelidna . }
$$

*347. P. мaritria, Bp. Tr. maritima, Brunn. Tr. nigricans, Mont. Tr. arquatella, Pall. Gould t. 334.

348. P. pectoralis, Say, Tr. pectoralis, Bp. Am. s. accid. Gould 1. 327.

Eur. s.

349. P. scurizi, Bp. Tr. schinzi, Bp. Am. Am.s.acc.Eur.s. Orn. Gould t. 33o.

*35o. P. crnclus, Cuv. Tr. cinclus et alpina, L. Boreal. tot. orb. Numenius variabilis, Bechst. G. t. 329. Afr. s. usque. d. Ancylocheilus, Kaup.

*35̃ i. P. subarguata, Cuv. Sc. subarquata el africana, Gm. Tr. falcinella, Pall. G.t. 328.

Eur. As. Afr. nee America.

170. Numenius, Lath. Gracticornis! Gray.

*352. N. Plaeopus, Lath. Sc. pllaeopus, L. Eur.As.etiamor. Gould t. 303. 
*353. N. тentrirostris, Vieill. Gould t. 304. Eur. m. As. Afr. *354. N. srquata, Lath. Sc. arquata, L. Eur. As. s. Af.s. Gould t. 3o2.

17I. Erolia, Vieill. Falcinellus; Cuv.

355. E. pYGMAEA, Gr. Sc.pygmaea, Gm.E.vaAfr. acc. Eur. ria, Vieill. $F$. cuvieri, Bp. Temm. pl. col. 510.

172. Calidnis, III. Arenaria, Mey.

*356. C. aretaria, Bp. Tr. arenaria, et Charadrius calidris, L。 Tr. tridactyla, Pall. C. tringoides, Vieill. Gould t. 335 .

Borcal. tot. orb. h. Ital. Casp. 173. TRINGa, L.

*357. T. canutus, L. Tr. islandica, Gm. Tr. Boreal. tot. orb. grisea et naevia, Gm. Tr. cinerea, Penn. Tr. rufa, Wils. Gould t. 324.

358. T. nupescbass, Vieill. Gould t. 326 . hyem. Afr.

Am. s. acc. Eur. occ.

174. MAChetes, Cur. Philomachus, Moehr.

*359. M. pugrax, Cuv. Tr. pugnax, L. Pa- Eur. univ. As.s. voncella pugnax, Leach, Gould t. 328. et occ. Afr. s.

175. Actitıs, Boie nec 111. Guinitta, (hinc Tringoides, Bp.) Gr.

36c. A. maculabies, Bp. Tr. macularia, L. Am. acc. Eur. $s$. Gould t. 317.

*36r. A. uypoleucus, Boie, Tr. liypoleucos, L. Eur. univ. As. Tr. leucoptera, Pall. Gould t. $3 \mathbf{1 6}$. etiam or.Afr.s.

176. Actiturus, Bp. Bartramia, Less.

362. A. bartramus, Bp. Tr. bartramia, Wils. Am.s.acc.Eur.s. Tr. longicauda', Nilss. Actitis bartramia, Naum. Gould t. 3 r3. 
177. Catoptropmonus, Bp. Irodites, Kaup.

363. C. semipalmatus, Bp. Scolopax semipalAm.s.acc. Eur.s. mata, L. Totanus crassirostris, Vicill. Gould t. 3ri.

178. Totanes, Bechst.

*364. T. roscus, Leisl. Tringa fiusca, L. Li- Eur. As. occ. mosa fusca, Br. Erythroscelus fuscus, Kaup, Gould t. 3og.

*365. T. calidnis, Bechst. Scolopax calidris, L. Tr. gambetta, Gm. Gambetta calidris, Kaup. Gould t. 3 Io.

*366. T. glareola, Temm. Tr. glareola et littorea, L. Rhyacophorus glareola, Kaup. Gould t. 315 . f. 2 .

*367. T. оснворus, Temm. Tringa ochropus, L. Helodromas ochropus, Kaup. G.t.3r5.f. r.

*368 T. stagnatilis, Bechst. Scolopax totanus, L. nec Auct. Tringa guinetta, Pall. Ilyornis stagnatilis, Kaup. Gould t. 314 .

179. Gцotтıs, Nilss.

*369. G. Canescens. Bp. Tot. glottis, Bechst. Sc. glottis, L. Lim. srisea, Br. Lim. glottis et totanus, Pall. Gl. chloropus, Nilss. Gould. t. 3r2.

180. LimosA, Br. Fedoa, Steph.

*3jo. L. rufs, Br. Sc. lapponica, L. Sc. leucopleaea, Lath. T. gregarius, Bechst. Gould t. 306 .

37 r. L. MEYERI, Leisler.

*372. L. aegocepha La, Bp. Sc. limosa et aegocephala, L. Lim. melanura, T'cmm. Eur. As. m. et or.

Eur. s. et occ.

Eur. As. etiam or.

Eur. Afr. s. As. eliam or.

Eur. As. eliam or. Afr. s.

Eur. As. Afr.s.

Eur. As. Afr. s. Boreal. orb. ant. h.Afr.s.usque: Gould t. 305 . 
181. Xencs, Kaup, Terekia, Bp. Simorhynchus, $K$. ot $\mathrm{Bl}$.

373. X. eirereus, Kaup. Sc. cinerea, Guldenst. Sc. terek, Lath. Lim. recurviroAs. Ocean. acc, stra, Pall. Tot. javanicus, Horsf. L. terek, Temm. L. indiana, Less. L. cinerea, K. et Bl. G. t. 307 .

\section{Famicia 55. Recurvirostridae.}

\section{Subfamilia 147. Recurvirostrinae.}

182. Recunvinostra, L. deocette, $B$ x

*374. R. avocetta, L. Gould t. 308 .

Eur. univ. As. occ. Afr. s.

183. Himaxtopus, Br. Iypsibates, Nitzsch.

*375. H. cardidus, Bonnat. Ch. limantopus, Eur. As. Afr.

L. H. vulgaris, Bechst. H. albicollis, Vieill. H. rufipes, Bechst. H. atropterus, Mey. Hyp. himantopus, Naum. Gould t. 289.

Familia 56. Pualaropodidae.

Subfamilia 1 48. Phalaropodinae.

184. Lobipes, Cuy. Phalaropus, Vieill.

*376. L. myperboneds, Cuv. Tringa hyperborea Boreal. tot. orb. et lobata, L. Tr. fusca, Grm. Pll. cinereus, $\mathrm{Br}$. Plo angustirostris, Naum. h. Eur. et As. Gould t. 336. med. 
185. Pinalaropus, Br. Crymophilus, Vieill.

*377. P. Fulicantus, Bp. Tr. fulicaria, L. Boreal. tot. arb. Tr. glacialis, $\mathrm{Gm}$. Ph. rufescens, Br. h. acc. Ital. P/. rufus, Bechst. P/h. platyrhynchus, Temm. G. t. 337 .

\section{Familia 57. Rallidae.}

Subfamilia 149. Rallinae.

186. Fueica, L.

*378. F. Cristata, Gm. Buff. pl. enl. 797 .

Afr. As. Hispan. acc.Gall. et Ital.

*379. F. Atra, L. F. aterrima, L. F. ae- Eur, As. Afr. thiops, Sparrm. Gould. 338.

187. Gallivuld, Br. Stagnicola, Brm.

\#38o. G. culoropds, Lath. Fulica chloropus, L. Eur. fere univ. Hydrogallina clıloropus, Lacep. G. t. 342.

As. occ. Afr. s.

188. Ponpiytrio, Br.

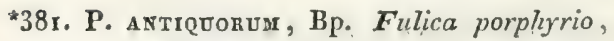
Eur. m. As, occ. Pall. P. hyacinthinus, Temm. G. t. 340 .

189. Ralles, L. Gallina, Ray.

*382. R. aquaticos, L. Gould t. 339.

Eur. univ. As. 8.

190. Ponzava, Vicill. Zapornia, Leach, Alecthelia, Sw.

*383. P. manuetta, Gr.' Rallus porzana, L. Eur. 'etiam bor. Ortygometra porzana, Steph. G. t. $343 . \quad$ As. s. Afr.

384. P. pygmaea, Bp. Crex pygmaea, Naum. Eur, occ. G. bailloni, Vieill. R. stellaris, Temm. Gould t. 344. 
*385. P. mrveta, Bp. $\boldsymbol{R}$. minutus, Pall. $\boldsymbol{R}$. pusillus, Gm. G. foljambei, Mont. G. t. 345 .

191. Ontygonetra, Ray, Grex, Bechst.

*386. O. crex, Gr. Rallus crex, L. Crex pratensis, Bechst. Gould t. 34 ז.

Eur.As.s.et occ. Afr.

\section{Familia 5g. Psophidat.}

\section{Subfamilia 154. Gruinae.}

Eur. praes. or. As. s.

192. Gnus, Pall. Megalomis, Gr.

387. G. Leucogerana, Pall. Ardea gigantea, Gm. Gould t. 27 r.

Eur. or. As, tota med. et s.

388. G. Axtigone, Pall. Ard. antigone, L. As. acc. Eur. or.

*389. G. cinerea, Bechst. Ard. grus, L. Grus Eur. As. Afr.s. vulgaris, Pall. Gould t. 270.

193. ANTnnopoldes, Vieill. Scops, Maehr. Tetrapteryx, Thunb.

*39o. A. vrkgo, Vieill. Ard. virgo, L. Gr. virgo, Pall. Scops virgo, Gr. G. t. 272.

I94. Balearica, Br. Grus, Gro

*39ז. B. paponisa, Vig. Ardea pavonina, L. Grus pavorina, Gr. Buff. pl. enl. 265.
As. m. Afr. s. Eur. or. ad m.

Afric. As. Ins. Mediterr. 


\title{
Sertio 2. Anscrucenc.
}

\author{
Familita 6o. Ardeidae. \\ Subfamilia 155 , Ardeinae.
}

195. ArdeA, L.

*392. A. ginenes, L. Ardea major, Gm. A. Eur. As.s. et or. cristata, Br. Gould t. 273.

*393. A. perpurea, L. Ardea caspica, Gm. Eur. As. Afr. Gould t. 274.

196. LGRETTA, Bp. Garietta, Kaup.

*394. E. alba, Bp. Ardea alba, L. Pall. A. Eur. or. m. As. candida, Br. Gould t. 276.

Afr.

*395? E. xantuodactyla, Bp. Ardea egrettoides, Term.

Sic.Dalm.Thrac. As. or.

*396. E. nivea, Bp. A. egretta, Rupp. nec L. Eur, m. ad or. $A$. orientalis, Gr. Ind. t. 65. E. nigri- As. m. rostris, $\mathrm{Bp}$.

*397. E. ganzetta, Bp. A. garzelta, L. Egrelta, Br. A. nivea, S. G. Gm. Hero-

Eur. pracs. or. dias garzetta, Boie. Gould t. 277.

As. eliam or.

197. Bupnus, Boie, Ardeola? Gr, Cancrophaqus? Kaup.

*398. B. russatus, Bp. $A$. russata, Wagl. $A$. aequinoctialis, Mont. A. bubulcus, Savgn, A. verany, Roux, Gould t. 278 .

*399. B. conatus, Bp. A. comata, Pall. A. ralAfr. As. m. et or. acc. Eur. loides, Scop. A. pumila, Lepech. A. Eur.m. et or.As. castanea, erythropus, squajotta, marsilii, Gm. G. t. 275 . 
*400. A. minuta, Bp. Ardea minuta, L. Ar- Eur. As. Afr. deola naevia, Br. Gould t. 282 .

199. Botaunus, Steph. Butor, Sr:

4or. B. mror, Bp. A minor, Wils. A. lentiginosa, Mont. A. mocoho, Wagl. G.t.28r.

Am. s. Accid. Britann.

\#402. B. stellaris, Boie. A. stellaris, L. G. Eur. As. s. ad t. 280 . occ.

200. Nxcticorax, Steph. Scotaeus, Bl. et $\boldsymbol{K}$.

*4o3. N. Griseus, Strickl. A. nycticorax, L. A. Eur. As. Afr. s. grisea, L. $N$. europaens, Steph. G. t. 279. occ.

\section{Subfamilia 56. Ciconinae.}

20I. Ciconia, Br.

*4o4. C. Nigra, Belon. Ardea nigra, L.C. Eur. orient. As. fusca, Br. Gould t. 284 .

Afr. s.

*405. C. AlBd, Belon. Ar. ciconia, L. G. t. 283 . Eur.As.occ.Afr 406. C. americana, Br. C. maguari, Temm. Amer. acc. Eur. Gould t. 285 .

\section{Familia 6i. Tantalidae.}

\section{Subfamilia 159 . Tantalinae.}

202. Inıs, Antiq. Cuv. Threschiomis, Gr.

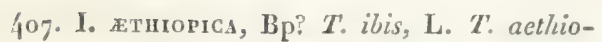
picus, Lath. Nunt. ibis, Pall. Ibis religiosa? Cuv.
Afr.b.As. occ. et finit. Eur. or. 
203. 'Legadis, Kaup, Falcinellus, Gr. nee Auct. Tantalides, Wagl.

*408. P. Falcinellus, Kaup. 'T. falcinellus, L. Eur. As. Afric.

$\boldsymbol{N}$. igneus? Gm. $\boldsymbol{N}$. viridis et castaneus, Ocean.

Br. I. falcinellus, Vieill. G. t. 3ог.

Familia 62. Piataleidae.

Subfamilia 60. Plataleinae.

204. Platalea, L. Platea, Br.

*409. P. leucorodia, L. Grould t. 286.

Eur. As. Afr.s.

Familia 63. Phaenicopteridae.

Subfamilia 161. Phaenicopterinae.

205. Phanicopterus, $\mathbf{L}$.

*4ro. P. roseus, Pall. Ph. ruber, L. part. Ph. Eur. m.praes.or. antiquorum, Temm. Gould t. 287 As. occ. Afr.s.

\section{ORDO VIII. ANSERES.}

Familia 64. Anatidae.

Subfamilia 162. Cygninae.

206. OLon, IVagl. Cygnus, Gr.

*4II. O. mansuetus, Wagl. $A$. olor, Gm. C. Eur. or. " sibilus, Pall. C. gibbus, Bechst. C. mansuetus, Ray, C. olor, III. Naum. t. $29^{5}$. G. t. $35 \%$.

4r2. O. пмметавиis, Bp. C. immutabilis, Eur.s. Yarr. III. p. х3г, fig. 
207. Craxus, Mley. Olor, Gr.

"413. C. musrcus, Bechst. A. cygnus, L. C. Eur. As.

ferus, Ray. C. olor, Pall. Olor ferus, Gr. C. melanorlynclus, Mey. C. xanthorlitnuts, Naum. t. 296 . G. t. 355 .

\{rf. C. mror, Pall. var. $\beta$. oloris, C. bewicki, Eur. s. As. s. Yarr. C. musicus? Fal,. C. islandicus, Br. C. melanorlinus, N. t. 297 . G. t. 356 .

\section{Subfamilia 163. Anserinae.}

\section{Crgropsis, Brandt.}

415. C. crgmordes, Brandt. Anas cygnoides, L. As. s. et finit. Anser cygnoides, Pall. Anser guineensis, Br. Buff. pl. enl. 346 .

4r6. C. canadexsis, Brandt, Anas canadensis, L. Buff. pl. enl. 346 .

Eur.or.

Am. s. Acc. Eur . boreal.

\section{Cuen, Boie.}

417. C. imperborea, Boic. Anas liyperborea, Boreal. tot. orl, Gm. A. nivalis, Forst. Anser lyperboreus, Pall. Gould t. 346.

\section{I0. Axser, Br.}

*418. A. enythropus, Flem. Anas erythropus, L. Anser albifrons, Bechst. Gould t. 349. Naum. t. 289 .

4rg. A. вrucir, Brm. A. albifrons, Fab. $A$. medius? Temm. jun. A. intermedius, Naum. t. 288.

420. A. brevirostris, Heck. A. brevirostris, et cineraceus, jun. Brm. A. minutus, Naum. t. 29 o.

Boreal. hyem. etiam Italia.

Eur. s. Island. Acc. Germ.

Eur.s.ad or.As. s. Acc. Germ. 


$$
20
$$

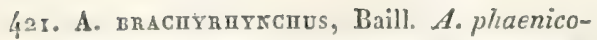
pus, Bartleti, $A$. brevirostris, Thienem. $A$. rufescens? Brm. Yarr. Br, birds fig.

Eur. s. Island. Scolia accid. Eur. med.

*422. A. Segetuar, Mey. Anas anser, L. Fn. Eur. As. S. A. segetum, $\mathrm{Gm}$. Ans. sylvestris, $\mathrm{Br}$. Gould t. 348.

*/23. A. cinereus, Mey. A. anser, L. Ans. vill- Eur. As. s. garis, Pall. $A$. pallustris, Flem. G. ¿. 347.

2II. Bernicla, Steph. Brenta; Br.

424. B. Ledcopsis, Stepl. Anas erythropus, Gm. Eur. As. Am? s. Anser bernicla, Pall. Gould t. 350.

*/25. B. ввеnta, Steph. Anas bernicla, Gm. Bor. tot. orb. A. torquata, Belon, Ans. Urenta, Pall. Anser torquatus, Fisch. Gould t. 352.

*426. B. Ruficollis, Steph. Anas torquata, Gm. Anser ruficollis, Pall. Gould t. 35 I.

Eur. s.pr. ad or. As. s.

$$
\text { 212. Plectroptenus, Leach, Anatigralla, Lafr. }
$$

427. P. Gambensis, Leach. Anas gambensis, L. Anser gambensis, Br. Latb. Syn. t. 102.

Afr. acc. Europ. etiam Ins.Brit.

\section{Cinenalopex, Steph.}

428. C. aegrptiaca, Steph. Anas aegyptia- Afr. Eur.or.m. $c a, \mathrm{Gm}$. Anser varius, Schn. G. t. 353 .

Acc. Ins. Brit.

\section{Subfamilia 164. Anatinae.}

214. TAdonns, Leach. Tulpanser, $K_{0}$ et $\mathrm{Bl}$.

*/29. T. rolpanser, Flem. A. tadorna, L. A. Eur. As. $s$. cornuta, Gm. $T$. bellonii, Steph. G. t. 357 .

215. Casarca, Bp.

*43o. C. rutila, Bp. Anas rutila, Pall. A.caAs. s. Eur. fere sarca, L. T. casarca, Stepl. G. t. 358. accid. 
216. AIx, Boie. Dendronessa, Sw. Lampronessa, Wagl. Cosmonessa, Faup.

43r? A. sponsa, Boie, Anas sponsa, L. Wils. Am. Orn. t. 70. fo 3.

Am.s.acc.Eur.ah, aiariis aufuga?

217. Maneca, Steph.Penelope, Antiq.

*432. MI. pexelope, Bp. Anas penelope, L. Eur. As. Mareca fistularis, Steph. Gould t. 359.

433. MI. americana, Steph. A. americana, L. Am. s. Acc. Ins. Wils. t. 69. f. 4.

Brit.

218. DAFiLA, Leach, Phasianurus, Wagl. Trachelonetta, Kaup.

*434. D. acuta, Leach. Anas acuta, L. A. cau- Eur. As. Am. s. dacuta, Pall. A. longicauda, Br. G. t. 365.

219. Qcerquedula, Steph. Tettion, Karip.

*435. Q. angustrrostris, Bp. Anas angustirostris, Ménétr. Anas marmorata, Temm. Daflla marmorata, Eyt. Gould t. 3,3.

436. Q. rormosa, Bp. A. formosa, Georg. A. As.s.Acc.Eur.or. torquata, Mess. A. picta, Stell. A. glocitans, Pall. nec Gm. Brandt. Ic. Ross. t. 4.

437. Q. вrimaculata, Bp. A. bimaculata, Penn. Britann. accid. A. glocitans, Gm. nec Pall. G. t. 363.

*/38. Q. crecca, Steph. Anas crecca, L. Boreal.antiq.orb. Gould t. 362 .

Afr. s.

220. Ptenocianea, Bp. Cyanoptemus, Eyt. Querquedula, Kaup.

*439. P. crncia, Bp. Anas querquedula, et Eur. As. Afr.s circia, L. Gould t. 364.

221. Rinvchaspis, Leach. Spatula, Boie; Clypeata, Brm.

\#44o. R. clyenata, Leach. Anas clypeata. I. Hemisph. arctic. Gould t. 360. 
222. Cihadelasius, Gir. Tinorhynchus, Eyt.

*44i. C. streperus, Gr. Anas strepera, L. Eur.As.s.Am.s. Gould t. 366.

\section{AvAS, L. Boschas, Sw.}

*442. A. вoscras, L. Anas adunca, L. monstr. Hemisph. bor. var. Gould t. 36r.

A. purpureo-viridis, Schinz. hybrida cum sequente.

224. Cairina, Flem. Moschatus, Less.

443. C. moschata, Flem. Anas moschata, L. As.occ.acc.Eur. Buff. pl. enl.

or. hosp. per

Eur. univ.

Subfamilia 65. Fuligulinae.

225. Somateria, Leach.

444. S. molissma, Leach. Anas mollissima, L. Anser lanuginosus, Br. Anias cuthberti, Pall. Gould t. 375 .

445. S. spectabilis, Leach. Anas spectabilis, L. Gould t. 375 .

Arctic. h. Eur. bor.

Arct.pr.As.hyem Eur. s.

226. OEDEMA, Flem. Melanetta, Boie.

a. Pelionetta, Kaup.

446. OE. perspicilata, Flem. A. perspicillata, Am.s.Acc.Eur.s. L. MIel. perspicillata, Boie, G. t. ${ }_{7} 7$.

b. MIelanetta, Boie. p.

*447. OE. fusca, Flem. Anas fusca, L. A. carbo, Pall. Melanetta fusca, Boie, G. t. 377 .

Bor, Eur. et As.

h. E. etian m. 
c. Oidemia, Flem. p.

*448. OE. wigra, Flem. Anas nigra, L. A. atra, Bor. Eur, et As. Pall. Mlelanetta nigra, Boie, G. t. 378. hyem. Eur.m. 227. Erismatura, Bp. Cerconectes, Wragl. Undina, Gould.

*449. E. levcocephata, Bp. Anas mersa, Pall. Eur. or. As. Ins. A. leucocephala, Scop. Gould t. $383 . \quad$ et litt. Med. 228. Braxts, Boie. Callichen, Brm. Netta, Kaup. Mergoides, Eyton.

*45o. B. rufina, Boie. Anas rufina, L. C. Eur. or. et m. ruficeps, Brm. $F$. rufina, B1. et. K. As, occ. G. t. 369 .

229. Arthis, Boie.

*45̆. A. Feriva, Gould. Anas ferina, L. Ful. Eur. As. bor. ferina, Bl. et K. Nyroca ferina, Gr. Gould t. 367 .

*452. A. marila, Bp. Anas marila, L. Fuli- Bor. h. etiam m. gula marila, Steph. Gould t. 3 7r. Eur. et As.

453. A. marnlordes, Bp. A. mariloides, Vig. Am. s. Acc. Ins. Yarr. Br. Birds III. p. 247. cum. fig. Britann.

230. Fulggord, Steph. Fultix, Sundev.

454. F. coltaris, Bp. Anas collaris, Donov. Am.s.Accid.Ins. A. fuligula, Wils. t. 147. A. rufitor- Britann. ques, Bp.

*455. F. cristata, Ray. Anas fuligula, L. Eur. As. eliam A. colymbis, Pall. Gould t. 3 go. maxim.orient.

23i. Nirnoca, Flem.

*456. N. Leucophthalira, Flem. $A$. nyroca, Eur. As. Afr. s. Guldenst. A. leucophthalma, Bechst. A. glaucion, Pall. Gould t. 368. 
232. StelleniA, Bp. (nec Stellaria) Eniconetta, Gr

457. S. dispar, Bp. Anas stelleri, Pall.A. dispar, Sparrm. A. beringi, Iath. Harelda stelleri, K. et $\mathrm{Bl}$. Gould t. $3_{72}$.
Ins. Am, et As. interm. Accid. Eur. $s$.

233. Glangula, Flem. Glaucion, Kaup:

*458. C. Glaucion, Boie. A. clangula et glaucion, L. A. hyemalis, Pall. Cl. vulgaris, Flem. Cl. clirysophthalma, Steph. Gould t. 379 .

459. C. islandica, Bp. A. islandica, Gm. $\mathrm{Cl}$. scapularis? Brm. Cl. barrowi, Sw. G.t.380.

Bor. orb. ant. h. Eur. etiam m.

46o. C. albeola, Jenyns, Anas albeola, L. Am. s. acc. Brit。 Wils. Am. Orn. t. 67, f. 2, m. 3. f.

234. Hanelda, Leach, Pagonetta, Käup.

46r. H. histrionica, K. et Bl. $A$. listrioni$c a$ et minuta, L. Cl. Jistrionica, Leach. Gould t. 38I.

*462. H. gracintis, Leach. A. glacialis el liyemalis, L. A. miclonia, Bodd. G. t. 382.

Bor. orb. tot. h. Eur. s.

Bor. orb. hyem. etiam Eur.m.

\title{
Subfamilia I66. Merginae.
}

\author{
235. Mengus, L. Merganser, $B r, p$,
}

*463. M. albelles, L. MI. minutus, L. MI. glacialis, Brunn. MI. stellatus, Br. G.t. 387 .

Mergus anatarus, Eimbeck. tab. in Isis. Acc. Germ.s. hybridus, cum Anati?

236. Mlèngavsen, Br. Mergus, $\boldsymbol{L}$. p.

464. MT. cuculcatus, Bp. Mergus cucullatus, L. Mrergans. virginianus cristatus, Br.G.t.386.

Am. s. Acc. Ins.

Brit. 


\section{$-5$}

*465. M. senRaton, Bp. Mergus serrator, L. Eur. As.s. Am s。 Merganser cristatus, Br. Gould t. 385.

*466. M. caston, Bp. Mergus castor et merganser, L. Gould t. 384.

Eur. As. s.etiam or. Am. s.

\section{Familia 65. Pelecanidae.}

\section{Subfamilia 169. Pelecaninae.}

237. TAcuypetes, Vieill. Atagen, Moehr. Fregata, Cuv.

467. T. Aqurus, Vieill. Pelecanus aquilus, L. $\boldsymbol{P}$. leucocephalus et palmerstoni, Lath. Buff. pl. enl. 508.
*468. P. carbo, Dumont, Pel. carbo, L. Carbo cormoranus, Mey. Halieus carbo, Ill. Hydrocorax carbo, Vieill. G. t. 407.

469. P. medrus, Nilss. C. cormoranus? Temm.

*470. P. graculus, Dum. P. graculus, L. Pall. nec Temm. Carbo crisiatus, Temm. pl. col. 322. Ph. desmaresti, Payr. Carbo desmaresti, Temm. G. t. $4 \mathbf{1} \mathbf{1}$.

*47r. P. cristatus, Dum. Pel. cristatus, Fabr. Br. nec Temm. Carbo graculus, Temm. Gould t. 4 ro.

472. P. prganaus, Dum. Pelecanus pjsmaeus, Pall. Gould t. 409 .

239. Pelecanus, L. Onocrotalus, Mtoehr.

*473. P. crispus, Bruch. P. onocrotalus a orientalis, L. p. P. onocrotalus, Pall. Eversm. Gould t. 406.

*/74. P. onocrotalus, Auct. $P$. onocrotalus a orientalis, L. $\boldsymbol{P}$.roseus, Eversm. G. 1.405.
Am. acc. Eur.s.

Weser I792.I.

Eur. s.

Eur. or. As. s. Ins. Medit.

Eur. bor. As, s.

As. Afr. Eur. or.

Eur. As.s. Am.b. Eur. bor. As, o. As. Afror. or.

As, occ. Eur. or.

Eur.nı.ador.As. occ. et m. Afr. 
240. Scla, Br. Morus, Fieill. Dypsome, Ill.

476. S. bassana, Br. Pelecanus bassanus et Mar. bor. hyem. maculatus, Gm. Sula alba, Hiey. Sula Eur. med. melanura, Temm. Gould t. 412. et 413 .

Familia 66. Laridae.

\section{Subfamilia I jo. Sterninae.}

24I. Srlochelinox, Brm. Hydroprogne, Kaup, Helopus, Hagl.

*477. S. Caspia , Brm. Sterna caspia, Lath. IIed. et m. orb. St. megarlyncha, Mey. Gould t. 4×4. ant. proad or.

242. Gelochelidon, Brm. Laropis, Wagl. Viralva, Steph.

*478. G. Axgrica, Brm. St. anglica, Mont. St. Eur. m. et med. aranea, Savi? nec Wils. St. affinis, Horsf. praesertim ad nec Ruppel. St. stubberica, Otto.G.t.4r6. or. As.

243. Thalasseus, Boie, Actochelidon, Kaup.

*479. T. cantracus, Boie. St. cantiaca, Gm. Eur.m. et med. St. striata, Gm. St. columbina, Schrank. St. boysii, Lath. St. canescens, Mey. Gould t. 415 .

48o. T. Afrisis, Bp. Sterna affinis, Rupp. Err. Mar. Graec. nec Horsf. St. media, Horsf. St. arabi- Indic. et Pacif. $c a$, Ehrenb. Rupp. Atl. t. I4.

\section{Sterxi, L. Iivundo, Ray.}

*48r. S. paradisea, Brunn. St.douglasi, Mont. Eur, med. et m. St. dougalli, Temm. Thalassea douglasi, Kaup, Gould t. $4 \mathrm{r} 8$.

*482. S. mrindo, L. St. fluviatilis, Naum. Eur. univ. As. Gould t. 417 . s. Afr. 
*483. S. macruna, Naum. St. arctica, Temm. St. hirundo, Fab. St. argentata, Brehm. Bor. extr. hyem. St. brachytarsa? Graba, Gould t. 419 . 484. S. Nirzscir, Kaup. Eur. etiam m. As. Afr. Am, s. 485. S. velox, Ruppel.

Eur. s.

As.Afr.acc.Eur. m. ad or.

245. Urdnochelidos, Boie, Viralva, Leach, Haliplana, Wagl.

a. Sternula, Boie.

*486. H. uгітта, Bp. Sterna minuta, L. St. parva, Penn. St. metopoleuca, Gm. Sternula minuta, Boie. Gould t. 420 .

b. Hydrochelidon, Brm.
*487. H. Ixтвida, Bp. St. Iyybrida, Pall. St. leucopareia, Natter. Pelodes leucopareia, Kaup, Gould t. 42ú.

*488. H. Ledcoptera, Boie. St. nigra, L. St. leucoptera, Temm. St. fissipes, Pall. Gould t. 423.

*489. H. Frssipes, Bp. St. fissipes, L. St. naevia, Br. Pall. St. nigra, Auct. Grould t. 422 .

246. Axots, Leach, Negalopterus, Boic.

49o. A. stonides, Gr. Sterna stolida, L. Gavia fusca, Br. Anous niger, Steph. IIegalopterus stolidus, Boie. G. t. $42 \mathrm{I}$.

\section{Sulfamilia 172. Larinae.}

247. Gavi, Boie, Pagophila, Kaup.

491. G. eburnea, Boie, L. eburneus, Gm. $\boldsymbol{L}$. niveus, Montag. $\boldsymbol{L}$. candidus, $\mathbf{P a b r}$. Gould t. 436.
Eur. et As.s.fere tot.dempt.extr. boreal.

Eur.m.praes.ad orient.As,occ. Afr. s.

Eur. m. et or. As. occ.

Tot. Hemisph. arctic.

Ocean. AtI. et Pac. fere univ. Acc. litt. Eur.
Circ. arct.hyem. Eur.modices. 
248. XEMA, Leach.

a. Xema, Eyton.

492. X. sabini, Leach, Larus sabini, Leach, Am.bor.acc. Ins. Gould t. 429.

Brit.

b. Chroecocephalus, Eyton, Gavia, Kaup.

* 49 3. X. minutum, Boie, Larus minutus, Pall. Eur. praes. or. L. atricilloides, Falkl. Hydrocoleus minuttes, Kaup, Gould t. 428.

*494. X. capistratum, Boie. Larus capistra- Eur. s. Med. tus, Temm. Bp. Fn. It. Av. t. 20.

*495. X. Ridibundum, Boie, Larus ridibundus, L. L. cinerarius et erythropus, $\mathrm{Gm}$. L. atricilla et naevius, Pall. G. t. 425 .

*496. X. Lambruschinm, Bp. Larus gelastes? Licht. Thienem. IK. et B1. L. letucoceplatus? Boissonn. L. cinerarius? Pall. $L$. roseus, Aliq. L. genei, Breme, L. tenuirostris, Temm. Icon. Fn. It. Av. t. 2 I.

$497^{\circ}$ X. plumbiceps, Bp. Larus plumbiceps, Temm. Xema caniceps, Brm.

*498. X. melanocephalum, Boie, Larus melanocephalus, Natt. Gould t. 427 .

499. X. Leucophthalium, Bp. Larus leucoplithalmus, Temm. pl. col. 366.

500. X. Atricilla, Bp. Larus atricilla, L. nec Pall. Gould t. 426.

5ог. X. іситнудтu, Bp. L. ichthyaetus, Pall. Ichthyiaetus pallasi, Kaup, Rupp. Atl. t. 17 .

249. Rissa, Leach, Cheimonea, Kaup.

*5o2. R. Tridactyea, Leach, Larus rissa et tridactylus, L. L. torquatus, gavia et canus, Pall. Laroides rissa, tridactyla et minor, Brm. Gould t. 435.

Eur. med. et m. As. s.

Medit. pracs. or. et m. M. rubr.

Medit. or.

Mediterr.

Medit. max. or. II. rubrum.

Am. s. Acc. Ins. Brit.

M. casp. M.rubr. Eur. max. or.

Circ.arct.h.Eur. etiam m. $\Delta \mathrm{fr}$. occ. 
503. R. noseA, Bp. Larus roseus, Jardine et Selby. Ill. t. I4. $L$. rossi, Sabin.

25I. LARts, L. Gavia, Boehr.

a. Laroides, Brm. Lams, Kaup.

${ }^{\sharp} 50$ 4. L. canus, L. L. liybernus, Gm. L. cyanorliynchus, Mey. Gould t. $43 \%$.

5o5. L. Cachinnays, Pall.

*506. L. audourvi, Payraud. Gould t. 438.

*507. L. Fuscus, L. L. flavipes, Mey. G. t. 43 I.

5o8. L. Levcopterus, Fab. L. islandicus, Edm. L. argentatus, Sabine. L. arcticus, Macg. L. glaucoides, Temm. Gould t. 433 .

5og. L. angentatus, Brunn. L. glaucus, Retz. Laroides major, argentutus, argenteus, argentatoiles et argentaceus, Brm. G. t. 434 .

b. Larus, Brm. Leucus, Eaup.

5ro. L. Guadcus, Brunn. Larus glacialis et giganteus, Benicke. $\boldsymbol{L}$. consul, Boie, $\boldsymbol{L}$. medius (hine minor), Brm. G. t. 432 .

*5ir. L. manivus, L. L. maximus, mulleri, fabricii et marinus, Brm. G. t. 430 .

\section{Subfamilia 173. Lestrinae.}

\section{Catarracta, Raj.}

512. C. SזUa, Brunn. Larus catarrliactes, L.

\section{Lestris catorrlactes, III. Gould t. 439 .}

Boreal. Am. s. Acc. Eur.

Eur. univ.

Mar. caspic. et rubr.Eur.or.m.

Cors. Ins. Hisp. Eur. univ.

Arct. hyem. Europ. $s$.

Eur. univ. As.s. etiam or.

Boreal. h. Eur. s. et med.

Eur.univ. Am.s.

Mar. bor. hyem.

Eur. med. 
253. LESTRYs, I1]. Stercorarius, Br. Catarracta, Brun.

*5з3. L. romanxa, Temm. Catarractes parasitica, var. camtschatica, Pall. Lestris

Bor. hyem.ctiam sphaeriuros, Brm. Gould t. 440.

*514. L. parasita, K.et B1. Cat. parasita, Brunn. Pall. Lestris richardsoni, Sw. G. 44r. Ital.

Mar. bor. hyem. etiam Ital.

515. L. cepius, K. et B1. Cat. ceplius, Brunn. Mar. arct. hyem. I. parasiticus, Sw. L. crepidata, Brm. Eur. med.

L. buffoni, Boie, Gould t. 442 .

\section{Familita 67. Proceliaridae.}

\section{Subfamilia 174. Procellarinae.}

254. DiouepeA, L. Albatmu, Br.

5i6. D. exulans, L. Buff. pl. enl. 237 .

Atl. et Pac. acc. Eur.

255. Procellaria, L. Wagellus, Ray, Fulmamus, Leach, Rhantistes, Kaup.

517. P. Gracialis, L. glacialis et hyemalis, Mar.arct.hyem. Brm. Gould t. 446 . Eur.vix.med.

256. Tualassidnoni, Vig. Ilydrobates, Boie, Procellaria, Kaup.

$$
\text { a. Oceanites, K. et Bl. }
$$

5 18. T. wruson , Bp. Pr. pelagica, Wils. Pr. oceanica, Licht. Hydr. oceaneus? Brm. Atlant. eur, afr. Audub. t. 270. am.

\section{b. Thalassidroma, K. et $\mathrm{Bl}$.}

519. T. zeachi, Bp. Pr. furcata? Gm. Pr. leaAtl. eur. afr. am. chi, Temm. Pr.bullocki, Selb. Hydr. leachii, Brm. Gould t. 447. f. I.

*520. T. pelagica, Vig. Pr. pelagica, L. Hydr. ferroensis et pelagicus, Brm. G. t. 447 . f. 2.

Mar. hemisph. arct. Medit. 
c. Bulweria, Bu.

52x. T. bulweri, Bp. Pr. bulweri, Jard. All. eur. praes. Gould t. 448 .

merid.

257. Puffinus, Br. Thiellus, Glog.

522. P. major, Fab. Puff. fuliginosus, Strickl. Boreal. Am. s. nec $\boldsymbol{P r}$. fuliginosa, L. jun.

*523. P. cinereus, Steph. Pr.putfinus, Temm. Mar. Eur.s. Menec L. Pro cinerea? Gn. Gould to $44^{5}$. diterr.

*52\%. P. Axgloncas, Ray, Pr. puffinus. L. Brunn. Pr. arcticus, Fab. Gould t. $44^{3}$.

Oc. eur. Medit. etiam orient.

*525. P. obscunde, Steph. Pr. obscura, Gm. Vieill. Gould t. 444.

Medit.Atl.praes. Americ.

Famila 68. Alcidae.

\title{
Subfamilia 175. Alcinae.
}

\author{
258. AlCA, L. Pinguinus, Bonn.
}

526. A. Impennis, L. Alca major, Br. G. t. 4oo. Mar. arctic.

259. Uramayia, Leach, Terda, Dumir.

*527. U. товda, Stepì. Alca torda et pica, L. Boreal. hyen. A. minor, Br. Gould t. for. Eur. m.

260. Mon.nox, Ill. Fratercula, Br. Larea, Vieill.

528. M. giacialis, Leach, Gould t. 404 .

*529. M. Arctices, Hil. A. arctica, L. II. fratercula, Temm. Lunda arctica, Pall. Fratercula arctica, Cuv. Gould t. 403.
Boreal.utr.Hem. Boreal.utr.Hem. Mediterr. 


\section{Subfamilia $\mathbf{1 7 7 . ~ U r i n a e . ~}$}

201. Mengoues, Ray, Arctica, Moehr.

3o. M. Alte, Alca alle, L. Uria minor, Br. Boreal. h. Eur.

Uria alle, Lath. Gould t. 402. med. Am. s.

262. Gnxlle, Brandt, Cepphus, Pall. Uria, Gr.

531. G. columba, Bp. Colymbus grylle, L. Ur. Mar. boreal. grylle, Lath. C. columba, Pall. C. grylle, Cuv. Grylle scapularis, Steph. Ur. groenlandica, Gr. Gould t. 399.

532. G. CARBO, Brandt.

Bor. Eur. or. As.

533. G. mandt, Bp. Uria mandti, Licht. Bor. Eur. et As.

263. UnIA, Br. Cataractes, Gr.

*534. U. romria, Brunn. Uria troile, Temm. Cepplus lomvia, Pall. Gould t. 396 .

Ocean.glac. Mar. s.Am.s. ad occ.

*535. U. AвRA, Pall. U. troile, Brunn, U. brunnichi, Sabine, $U$. francsi, Leach, Alca pica, Faber. Cepphus arra, Pall. G. t. $39^{8}$.

536. U. nungvis, Brunn. $\boldsymbol{U}$. lacrymans, La Py- Islandia. lage, U. leucopsis, Brm. Gould t. 397.

\section{Familia 69. Colymbidae.}

\section{Subfamilia 1 78. Colymbinae.}

264. Colтsвus, L. Cepphus, Pall. Eudytes, $M$.

\#537. C. Glacialts, L. C. torquata, Brunn.

C. immer, Brunn. L. Gould t. 393 .

*538. C. Ancticos, L. Cepphus arcticus, Pall. Gould t. 394 .

539. C. BaLtaricus, Hornschucb, C. macrorliynchus? Br.
Eur. praes. s. $\Lambda$ s.

s. Am. s.

Eur. praes. s. As. s.

Eur. septentr. 
*54o. C. septentrionatis, L. C. Zumme, Brunn. Boreal. h. etian Cepphus septentrionalis, Pall. G.t. $39^{5}$. Eur. merid.

\section{Subfamilia I79. Podicipinae.}

265. Popiceps, Lath. Colymbus, Br.

*54 ז. P. CRistazers, Lath. Col. cristatus, L. Eur. As. s. Afr. C. cornutus, Br. Pall. C. urinator, Scop. Lophaythyia cristata, Kaup. G. t. 388.

*542. P. Longrnostris, Bp. Compl. Faun. It. t. Sardinia Insula.

*543. P. subcristatus, Jard. Col. subcristatus, Eur. As.s. etiam Jacq. $\boldsymbol{P}$, rubricollis, Lath. C. cucullatus ad or. Am. s. et naevius, Pall. Pedeaithyia subcristala, Kaup, Gould t. 389.

544. F. Arcticus, Boie. Naum. t. 7r. f. 109. Eur. septentr.

*545. P. Connutus, Lath. Col. cornutus et ob- Memispl. bor. scurus, L. Dytes cornutus, Kaup. G. t.39o.

*546. P. Adritus, Lath. Col. auritus, Br. Eur. As. s. occ. Proctopus auritus, Kaup, Gould t. $39 \mathrm{r}$. Am. s.

*547. P. Mzvon, Lath. (minime Sylleocyclus Bp. Eur. univ. Afr. err. txp.) Col. fluviatilis, Br, Col. mizor et hebridicus, L. Gould t. 392 . 
Non ho incluso in questo Cataloso i srguenti Uccelli perchi non li credo europei scbbrne altri li ritenessero tali, ed io stesso per alcuni fossi dello stesso parere.

I. Sxlyia antuoides, Vieill. ( pl. enl. 752 . f. 1.) che sembra la Motacilla noveloracensis, Gm. dell' America settentrionale.

2. Cinclus pallasi, Temm. specie asiatica data all' Europa per error di geografia.

3. Turues nuerus, Bechst. specie reramente dubbia, e se diversa dalle registrate abita esclusiramente $\mathbf{l}^{2}$ Asia seltentrionale.

4. Mruus Rufus, L. specie americana che si dice talvolta uccisa in Irlanda.

5. Oreocracla varios, Gould (Turdus varius, Horsf.) che dalla Nuora Olanda crediamo a stento emigri nel Giappone, ma per niun modo possiamo ammettere a formare una seconda specie europea del genere.

6. Aladda minaculata, Ménétr. specie non mai finora osservata dentro i limiti Europei comunquemente si voglia allargarli.

7. Corrus dacrices, Pall. buona specie dell' Asia d'onde ha sconfinato soltanto per quei naturalisti che l' hanno scambiata con una varietå del Corvus monedula.

8. Syrnuaptrs paranoxu's, 111 . che sembra non uscire dalla Siberia. 
9. Lagarus nepestris. Sabine, seppur buena specic. esclusiramente americana.

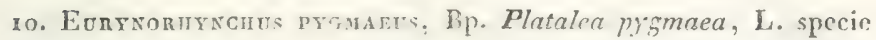
Asiatica, per falsa asserzione registrata fra le Europee.

Ir. Andea herodis, L. specie americana che non crediamo mai volata in Europa.

Piuttosto che ammellere come specie d'Europa le antecedenti ed altre o equivocate, o supposte, o ammesse per induzione di vicinanza geografica, o perchè portaieri dall'uomo, o almeno da qualche bastimento su cui ricorrarono in tempesta; sarei in verita tentato di eliminare le specie seguenti.

I. Hatiates reccocenelues, Sargn. Aquila d'America, circa la quale vorrei fosse provato che gli esemplari di Norregia spettassero veramente ad essa e nun ad una varietà che a cagione del clima abbia il capo piü bianco dell' Halraetus albicrlea.

2. Nauclerus forcatus, Vig. cui malgrado le potentissime ali, appena vorremo credere europeo per li due esemplari accidentalmente veduti nella Gran Brettagna nel 1772 e 1805 .

3. Elanes melayopterus, Leach, uccello africano che tuttaria non si è mostrato che nel centro di Europa, onde si potrebbe sospettare fuggito da cattività.

4. Falco coxcolor, Temm. pl. col.33o ugualmente d'Africa, che può essere stato scambiato col Falco eleonorae da chi lo disse europeo.

5. Caprmulgus chmacenus, Vieill. venuto dall Africa una sola volta in Provenza.

6. Turdus iuroreus, Pall. il quale, se raramente dalia costa oc- 


\section{6}

cidentale di America trapassa nella Siberia più orientale, appena può supporsi che tocchi mai i limiti europei.

7. Tundus mron, Gm. specie americana, che per essere stata presa parechie volte in Italia, in Germania, in Inghilterra, non è a credere sia uscita di gabbia; quantunque inesplicabile paja che i suoi affinissimi non emigrino come essa in Europa.

8. Tundus maratorius, L. comunissimo in America, ma forse troppo accidentale in Europa.

9. Parus bicolor, L. dell'America settentrionale, e comune in Groenlandia; onde forse registrato come uccello della monarchia Danese, 'e quindi europeo.

10. Struturs uyemalis, Bp. Emberiza hyemalis, L. specic ammessa europea come la precedente a cagione senz' altro deila stessa geografica estensione pólilica.

II. Cureus, meglio peró Coccyzes americanos, Vicill. uccello americano, quantunque più volte ucciso in Iŕlanda.

12. Ectoristes migratorius, Siv. che dubitiamo forte non esservi altrimenti che portato.

13. Ortyx vinginiana, Bp. affatto indigena di America, ma inclusa nel nostro catalogo, per trovarsi del tutto naturalizzata in varie parti d'Europa e specialmente in Inghilterra.

14. Otis поuвана, Gm. troppo accidentale dall'Africa.

15. Ciconia anericara, Br. Accidentalissima dall'America, e quello che è più dalla meridionale, onde la crediamo sfuggita.

16. Botaurus lentiginosus, Montag. certamente identico col Botaurus minor, Wils. d'America, d'onde s'ignora come venisse una sol volta in Inghilterra. 
17. Dromedea exurans, L. Le cui ali benché robustigsime appena possono persuadere qualche lunga rolata dai tropici fino alla fredda Norveggia ove soltanto fu vista.

18. TachYpetes ąuruus, Vieill. Lo stesso dicasi di quest'americana specie veduta una sol volta alle foci del Weser nel gennajo del 1772 .

19. Crgnopsis caradersis, Brandt, quantunque uecisa piu rolte nelle Isole Brittanniche.

20. Phectropterus gajinexsis, Leach, specie áfricana.

2i. Cremaropex aegretiaca, Steph. come la precedente.

22. Arx spossa, Boie, bellissima specie americana.

23. Camrasa moschata, Flem. forse sfuggita da cattivili. 


\section{Catalogo Metodico degli Uccelli Europer.}

(Continuazione e fune, vedi T. VIII. p. 425)

NOTE CHF ESPONGONO I CARATTERT DISTINTIVI DELLE FATIGLIE E SOTTOFATIGLIE NOVELLAMTENTE AGGUUNTE NELLO SPECCHIO GENERALE DTL SISTEMA ORNITOLOGICO.

\section{F A MILIAE。}

(A) по. Todida. Rostrum longulum, rectum, latum, apice inflexo: pedes digitis saltem externis conjunctis: alae breviculae: cauda brevis .

(B) г5. Promeroprd.e. Upupidas, in quibus subfamiliae Upupinarum et Promeropinarum adscribebantur, distinguo nunc in Promeropidas et Upupidas ob masimam intercedentem differentiam pedum et morum, familiarum gradu collato .

(C) i8. Caeraebidae, loco tantum Cymiridarum, amplectentes Nectarininas et Caeraebinas.

(D) 20 Menuridae. Colligit Familia haec plures alibi longeque dispersas aves inter se affines. Rostrum modicum, subemarginatum: pedes robusti, digitis anticis ad basim adnatis: alae breves, rotundatae: cauda erectilis. 
(E) 45. Megaponimae. Rostrum lweve, a hasi incurvum: pedes robusti, pracgrandes; digitis anticis subaequalibus: alae breves.

(F) 5r. Dididae. Familia recenter exciacta. Monumenta indubia rostrum ac pes in Nusaco Ashmoleano Osoniensi admonent extitisse reapse avem, cui nomen Didus ineptus, L. hic collocandam . Rostrum breve, robustum, vulturinum : pedes ecalcarati.

(G) 53. Aptergutdae. Charactercs Subfamiliaa Apteryginarum: Rostrum elongatum, gracile, grallaceum : pedes calcarati.

(II) 58. Palamedemae. Familia hace constat aliquibus Rallidas inter et Psoplididas adhuc receptis avibus. Rnstrum breve, subfornicatum : digiti longissimi: alae calcaratae.

(I) 62. Pentaleidae. Habeué characteres Plataleinctum subfamiliae ab Ardeidis ablatae, Phaenicopteridis adpropinquae.

\section{SUBF A III A E.}

(a) 3. Platycercinas. A Pezoporinis fere grallatricibus valde distinctae praecipue in moribus. Cauda in utrisque longa, cuneata; sed rectrices quae in Platycercinis latissimae et rotundatae, in Pezoporinis angustae acutacque sunt: alae in primis breves, in alteris elongatae.

(b) 8. Cathartinae. Nares perviae: pollex brevis, elevatus; quando in Vulturinis nares imperviae: pollex bene explicatus, imus.

(c) so. Gypohieracinac. Synonymae Racaminarum, Gray. 
(d) 3o. Steatornithinae. A Caprimulgidis detractae, quia omnino baccivorac, minime insectivorae, sub Coraciadidis provisorie militant, quibus adnumerandae potius essent Oriolinae, Turdidis ablatae, nisi conjungantur Sturninis.

(e) 32. Todinae. Rostrum elongatum, naviculare, apice abrupte angustato: pedes exiles, longi, digitis omnibus brevibus, lateralibus inaequalibus, ad basim plus minus connatis: alae brevissimae; rotundae : cauda brevissima, arcta, rotundata .

(f) 38. Promeropinae. Earum characteres praecipui Familiam distinguunt Promeropidarum.

(g) 41. Phaetornithinae. Rostrum plus minus curvatum, compressum : cauda longa, gradata.

(h) 42. Lampornithinae. Rostrum rectulum, depressum: cauda brevis, integra; quando in Trochilinis. Rostrum rectum vel curvatum, cylindraceum: cauda modica, furcata, vel saltem emarginata .

(i) 45. Melithreptinae. Eadem ac Manorhininarum subfamilia alterius editionis. Rostrum breve, validulum, rectulum, compressum : cauda truncata.

(k) 46. Meliphaginac. Rostrum modicum: cauda longa, cuneata, vel saltem rotundata.

(1) 47. Myzomelinae. Rostrum longum, debile, maxillis incurvis: cauda brevis, truncata.

(m) 48. Menurinae. Rostrum modicum, rectum, ad basim depressum: nares nudae, submediae: pedes praegrandes, robustissimi, digitis anticis subaequalibus, unguibus maximis, sub- 


\section{$9^{1}$}

rectis, obtusis: alae breves: cauda longa, mirabiliter se explicante.

(n) 49. Malurinae. Rostrum breve, subulatum: nares basilares, membrana semiclausae : pedes grandes, digitis anticis inaequalibus: alae valde rotundatae: cauda elongata, gradata, plus minus rigida.

(o) 5o. Troglodytinae. Eadem ac Thryothorinarum subfamilia, ripristinato ayi hoc nomine non opportuno generi Simiae Mammalium. Rostrum longulum, subincurvum, compressum : nares basilares membrana subfornicatae : pedes grandes, digitis anticis inaequalibıs : alae rotundatae : cauda rotundata vel gradata, brevicula, mollis .

(p) 53. Furnarinae. Rostrum basi plumosa, elongatum, compressum, plus minus incurvum, marginibus ad medium inflexis : rictus glaber: nares grandes, depressae, nudae: pedes robusti, incessores, digito postico longissimo: alae breves, rotundatae: cauda lata, rectricibus mollibus, quamvis acutis.

(q) 55. Dendrocolaptinae. Rostrum in variis varium : pedes breves, scandentes, digitis externis aequalibus ad primum usque articulum connexis, interno brevissimo, sublibero; postico aequali, ungue majori : cauda cuneata, rigidissima, rachidibus prolongatis, corneis, acutis.

(r) 6o. Pytilinae. Subfamilia haec melius sub Tanagridis militat ob rostrum emarginatum et trigonum, naribus approximatis, quam sub Fringillidis. Phytotominae vero, quae sequuntur, perperam sive cum Tanagridis sive cum Fringillidis conjunctae, erigendae vel sunt 
in Familiam, vel inter Colidas adnumeran= dae, ob tarsos postice reticulatos.

(s) 63. Anthinae. Pedes graciles: alae modicae, remigibus primis quacuor subaequalibus, teriariis elongatis, obtusis: cauda modica, emarginata: colores Alaudarum. In Motacillinis vero, pedes robusti: alae acutae: cauda longa, divaricata aut truncata: colores Picarum.

$\left.{ }^{*}\right)$ 8o. Ampelidinae. Familiam 28. Ampelididarum distulimus hue ob perspicuam affinitatem cum Turdidis, Muscicapidis, Lanic'is. Ampelidinarum subfamilium posuinus loco Bombycillinarum, Cotinginas loco Ampelidinarum, restitutis cuique nominibus legitimis.

(t) 98 . Geospizinac. IIanc constituimus Subfamiliam ad eos dumtaxat colligendos Americae Fringillidas, qui pedibus instructi rasoribus solo gaudent more gallizaceo, quaecumque sit forma rostri in iisdem.

(u) г 05. Phaenicophacinac. Rostrum ingens, crassum, laeve; nares ad basim in sulco, ovatae, imae, setis rigidis protectae; facies nuda.

(v) ro8 Centropinae. Rostrum robustum. Pedes tarso aequali digito nedio; unguibus anticis gracilibus, subincurvis; postico longissimo, subrecto .

(x) I 12. Colaptidinae. Plures ab auctoribus recentioribus propositas respuendo subfamilias Picidarum duas tantum addimus binis per nos constitutis, Colaptinas nempe intermedias Yunginis et Picinis, non secus ac Picumnincs intermedias Picinis et Bucconidis. Rostrum curvum, compressum, carina laterali fere nulla, latius quam a'tum ad loasim; nares plumis semitectae. 


\section{$9^{3}$}

(y) 1:4. Picunininie. Rostrum breve, rectum, compressun, carina nulla: nares plumis semitectae: alae rotundatae, remigibus gradatis: cauda brevis, gradata, rectricibus mollibus. In Yunginis cauda longa, lata, subtruncata.

(z) 120. Opisthocominac. Fnmilia fortasse distinctissima. Rostrum breve, crassum, robustum, convesum, lateribus compressis, ad basim abrupte dilatatis; mandibula forti, subtus angulata: nares medianae, membranaceae : pedes robusti, musculosi; tarsi reticulati digito medio breviores; digiti lnterales elongati, aequales, sejuncti usque ad basim; plantae complanatae; digitus postirus subfimbriatus: alae modicae, remigum prima lorerissima, insequentes quatuor gradatae, sexta longissima omnium : cauda longe, rotundata, rectricibus decem.

(ai) 121 . T'reroninac. Rostrum breve, robustum: tarsus brevis, plumosus.

(b1) 123. Gourinae. Eaden a: Fylopninarum subfamilia, nomine ripristinato.

(cc) 12S Talegaillinae. Resíum crúsum: nares basales, membranaceae, foro late rotundo: caput subauâun: caudà lata, zectricibus is.

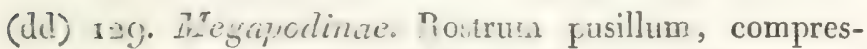
sum: nares medicicaè, stuçae : caput plumosum: cauda brovissima.

(ce), т 3r. Ci'acina?. Digiti lnterales, inaequales: quaudo in Penclopiris aequales.

(*) I’o. Tumicinac, quae prins Onygidinae, TetraoNidis potius adscribinus, natura duce, quamvis inter Crypturidas adnumerares si ad anomalos pedes respicias. 


\section{4}

(fi) 1 43. Vanellinae. Tarsi scutellati alae elongatae, latae, remigibus quatuor primis subaequalibus; quando in Charadrinis; tarsi reticulati: alae longae, angustae, prima remigum longissima omnium.

(gg) i 50 . Araminae. Contrectando iterum Graliarum Ordinem, eumque in Gallinaceas ac Anseraceas dividendo, placuit genus Arami Ardeidis Rallidisque onnino medium in subfamiliam redigere. Rostrum elongatum, gracile, rectum, subcylindraceum, inferius subturgescens, prope apicem subinflexum; nares in imo longoque sulco: pedes longissimi; digitis omnibus praelongis, vel parum palmatis, vel nihil: alae longulae, rotundatae.

(hh) 153. Psophinae. Recipit subfamilia haec Psophidas a Gruinis diversas, eique nomen conveniens damus, Palamedeinis in familiam elevatis. Rostrum breve, compressum, fornicatum, vere gallinaceum: alae breves, concavae: cauda brevissima. Quando in Gruinis rostrum rectum, ardeaceum: cauda longula remigibus secundariis recumbentibus tecta.

(ii) 158. Dromadinae. Rostrum robustum, rectum, compressum, basi depressa, subtus valde angulatum: pedes graciles, compressi, digitis anticis in basi palmatis, postico imo, elongato: ungues depressi: alae modicae, acutae.

(kk) 167. Heliornithinae. Anomalia pedum in generibus Heliornis et Podoa a caeteris Pelecanidis, satis nobis nunc est, ut ea in peculiari subfamilia conscribamus, dum ab Auctoribus quibusdam, hac punctim de causa, alibi, et extra ordinem, ac vel inter Rallidas collocantur. 


$$
9^{5}
$$

Rostrum conico-elongatım, culmine subincurvo, apice emarginato: pedes digitis partim palmatis: alae modicae: cauda ampla, rotundata.

(11) 177. Urinae, Rostrum longulum, gracile, hinc inde plumosum usque ad nares: pedes graciles, tridactyli. Spectant ad familiam Alcidarum praeponendam Colymbidis, quae constant tantum Colymbinis et Podicipinis, optime collimantibus cum Familia Splecniscidarum. 


\section{ADDENDA ET CORRIGENDA.}

Species 6. Gypaetus barbatus, Cuv. Optimam non habet speciem in var. orientali, prout errantes typi contradictorie exhibent, sed in G. MERIDIONALI.

Species I1. Aquila peniata, Cuv. *asterulo donanda quia itala. Capta prope Senas visitur in earumdem Musaeo, cujus auctor benemerentissimus Abbas Baldacconi.

Species 83. Parus cyanus, Pall. deme asterulum.

Species 228. Emerriza aureola, Pall. deme asterulum quia minime inter italas aves adnumeranda, non secus ac praecedens.

Species 233. Emberiza rithyornus, Pall. *asterulo potius donanda.

Species 239. Emireniza striolata, Cretzschm. Fringilla striolata, Licht. Rupp. Atl. Afr. tab. Io. fig. a.

Species 259. Serrinus islandicus, Bp. asterulum deme.

Species 264. Linota donealis, est Fringilla borealis, Vieill. nec non Temm., posthabitis suppositis distinctionibus, et Linaria canescens, Gould. Sed quia est etiam Fr. linaria, L. legatur ideo Linota Linaria, Bp., dum species 263. Fringilla linaria, Temm. hujus nominis usurpatrix, appellanda est Linota rufescens, quae minor cum sit rufescit ob limbos tectricum fascias alares constituentes, dum L. linaria quae major, canescit ols albidos tectricum limbos, et 
praecipue iu uropsgio. Utimur generico nomine Liмота, rejectis Brelimianis Linaria et Cannabina, perperam synonyma apud cl. Gray, quia botanica sunt.

Species 339. Galdinago moxtıgut, Bp. eadem ac Gal. linago major, cui substituenda fortasse G. PEREGrina, Baillon.

Species 520. Thalassidroma pelagica, Vig. Avis haec cum indubie sit typus generis Procellariae, L. species immo unica in editionibus Linneanis primis, genus ideo Thalassidroma donandum prorsus est nomine Procellariae. Idcirco ubi reperias $T h$. pelagica legas $P$. pelagica, L.; ac loco Th. wilsoni ponas $P$. wilsoni, Bp. Legas etiam $P$. leachi, Temm. loco Th. leachi; legas quoque $P$. bulweri, Jard. loco Th. bulweri. Procellariae dehinc nomen a grandibus albidisque Procellarinis usurpatum facile permutaremus in Fulmardm, Leach. potius quam cum illegitimo TVagello, Ray. Idcirco loco $P$. glacialis legas $F$. glacialis, Leach. 









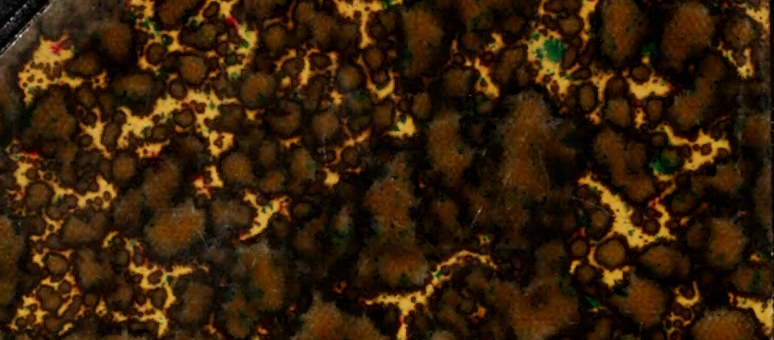

1. $5 \sin ^{3}$ is

C. 15

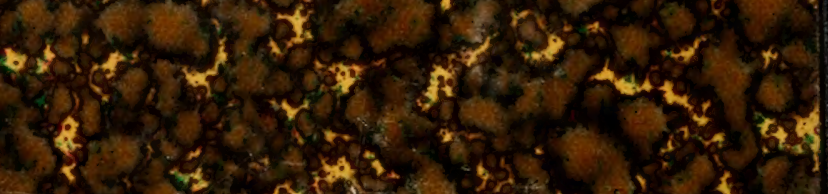

3. $\left.2 x^{2}\right)$

18. 2.

(3)

a.

$x^{2} \rightarrow \infty$

ba

$\log _{\text {करे }}$ ?

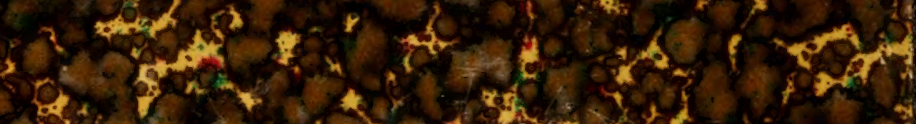

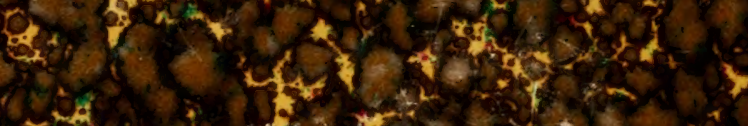

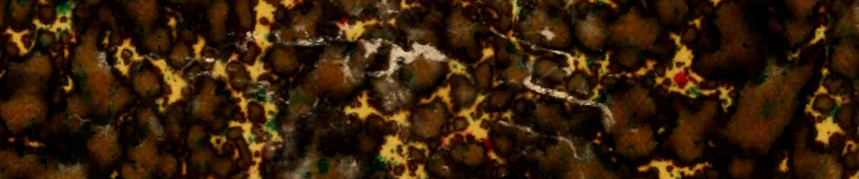

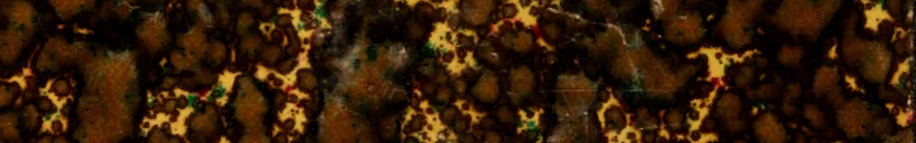

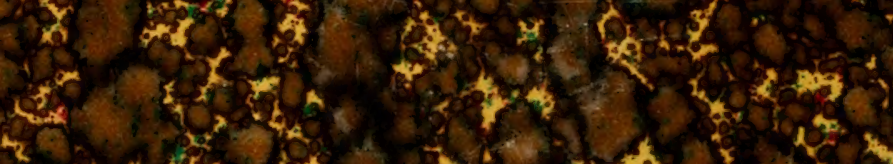

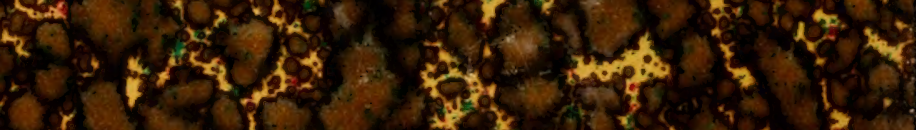

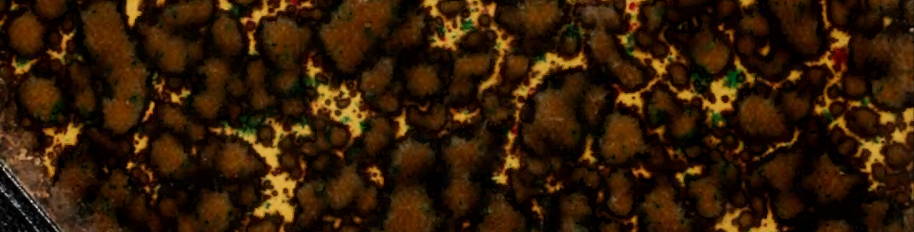

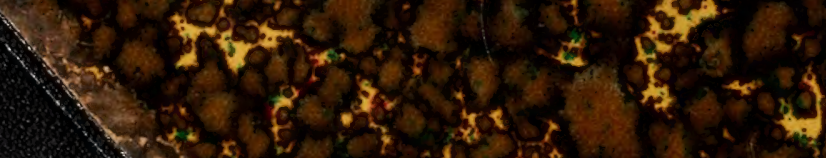

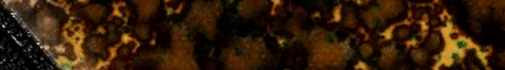

